\title{
Conductas, Estrategias y Rendimiento en Lectura en PISA: Análisis para el Perú
}

\author{
Behaviors, Strategies and Achievement in Reading \\ Comprehension in PISA: Analysis for Peru
}

\author{
Santiago Cueto ${ }^{1 *}$ \\ Juan León ${ }^{1}$ \\ Ismael G. Muñoz ${ }^{2}$ \\ Elizabeth Rosales ${ }^{3}$
}

${ }^{1}$ GRADE ${ }^{2}$ Universidad Estatal de Pennsylvania ${ }^{3}$ Universidad de Toronto

\begin{abstract}
$\mathrm{El}$ aprendizaje de la lectura es considerado internacionalmente un objetivo central de la escolaridad. En el presente análisis usamos los datos de PISA en lectura para determinar si las características socioeconómicas de los estudiantes se asocian con su rendimiento, conductas y estrategias educativas, así como su entorno escolar. En cuanto a los recursos educativos de los estudiantes en sus instituciones y hogar, se observó una gradiente positiva asociada al nivel socioeconómico. Si bien el rendimiento promedio en lectura de los estudiantes peruanos creció notablemente entre el 2000 y 2009, son los estudiantes de menor nivel socioeconómico los que menos mejoraron. Encontramos tres variables pedagógicas con alto peso explicativo en el rendimiento en lectura: estrategias de comprensión, de síntesis y de memorización (las dos primeras en positivo y la última, negativo). Una fuerte asociación entre el clima del aula y el rendimiento en lectura, en particular en las instituciones educativas de bajo nivel socioeconómico. Los resultados sugieren que los estudiantes de menor nivel socioeconómico tienen menores oportunidades de aprendizaje y menor rendimiento. Por ello se recomienda priorizar la atención e inversión en las instituciones educativas que atienden a estos estudiantes y diseñar y evaluar el impacto de intervenciones centradas en las variables pedagógicas.
\end{abstract}

Descriptores: Lectura, Evaluación del estudiante, Rendimiento escolar, Estrategias educativas, Educación formal, Perú, Nivel socioeconómico.

Learning to read is internationally considered a central goal of schooling. In this analysis we used data from PISA on reading comprehension for Peru, with the aim of determining if the socioeconomic characteristics of students are associated with their performance, behaviors and educational strategies, as well as with their school environment. Regarding educational resources for students in their homes and institutions, a positive gradient was found, which was associated with socioeconomic status. While the average reading performance of Peruvian students grew significantly between 2000 and 2009, students of lower socioeconomic status showed the lowest improvement. There are three pedagogical variables with high explanatory power in reading achievement: comprehension, synthesis and memory strategies (the first two with a positive sign, and the last one with a negative sign). We also found a strong association between classroom climate and reading performance in educational institutions of lower socioeconomic status. Our results suggest that students with lower socioeconomic status have lower levels of learning opportunities and lower performance. We suggest attention and investment in the educational institutions these students attend, as well as designing and evaluating the impact of interventions focusing on the pedagogical variables mentioned above.

Keywords: Reading, Student evaluation, Academic achievement, Educational strategies, Formal education, Peru, Socioeconomic status.

*Contacto: scueto@grade.org.pe

ISSN: 1696-4713

www.rinace.net/reice/

revistas.uam.es/reice
Recibido: $\quad 1$ de enero 2016

$1^{\text {a }}$ Evaluación: 1 de marzo 2016

$2^{\text {a }}$ Evaluación: 6 de mayo 2016

Aceptado: $\quad 9$ de junio 2016 


\section{Introducción}

El presente estudio tiene como objetivo analizar la asociación entre tres grupos de variables: los resultados en la prueba PISA de lectura, administrada el 20091, las características socioeconómicas y patrones de conducta lectora de los estudiantes, y las características de las instituciones educativas a las que estos asisten, incluyendo las prácticas de sus docentes. Si bien existen diversos estudios en América Latina vinculando nivel socioeconómico y rendimiento, existen relativamente pocos vinculando estos simultáneamente con las otras variables mencionadas antes, particularmente en el campo de la lectura en secundaria. En el análisis, el enfoque subyacente es de equidad y oportunidades de aprendizaje. El tema de equidad se aborda en tanto que históricamente las pruebas de rendimiento en el Perú han mostrado notables brechas entre grupos, asociadas principalmente a pobreza, ruralidad y etnicidad (entre poblaciones indígenas y castellano hablantes). Como lo sugiere Tiana Ferrer (2011) es importante ir más allá de la superficie de los datos PISA y proponer análisis que ayuden a conocer nuestros sistemas educativos con el fin de proponer líneas de mejora. Consideramos que el presente análisis de los datos PISA busca contribuir a las mejoras del sistema educativo peruano y otros con similares niveles de desarrollo al proponer un análisis con enfoque de equidad, usar datos del primer ciclo completo de evaluación de lectura de PISA (2000 y 2009), y analizar variables que podrían ser influenciadas por el sistema educativo. A continuación una breve revisión de estudios vinculados al aprendizaje de la lectura, con énfasis en el desarrollo de esta habilidad durante la secundaria.

\section{Antecedentes}

\subsection{El aprendizaje de habilidades en Lectura}

Junto al aprendizaje de la matemática, el de comprensión de lectura es ampliamente considerado un resultado de la escolaridad a la vez que un instrumento para futuros aprendizajes. Como respaldo de la relevancia que se le asigna internacionalmente se puede mencionar que en todos los países de América Latina que evalúan de manera estandarizada a sus estudiantes a escala nacional se incluyen evaluaciones de habilidades de lectura (Ferrer, 2006).

En este contexto resulta relevante preguntar cómo se adquieren habilidades en lectura, y luego cómo estas se pueden profundizar a lo largo de la vida. En los Estados Unidos en la década del 90 el Panel Nacional de Lectura, formado por expertos en el tema, hizo una serie de recomendaciones ${ }^{2}$. En esta se sugiere enfatizar 5 componentes: conocimientos del alfabeto (nombres de letras y sonidos), conciencia de fonemas (correspondencia de sonidos escuchados y unidades fonológicas mínimas, como por ejemplo letras), fluidez (lectura de manera rápida, precisa y entonada; Abdazi, 2010), vocabulario y comprensión (Paris, Morrison y Miller, 2006). Si bien el logro en un área está relacionado a las otras, esto no implica que con impulsar el aprendizaje de cualquiera se puedan lograr las demás, sino que se sugiere que la enseñanza de la lectura se debería planificar con un enfoque balanceado entre componentes. Los dos primeros y en alguna medida el tercero

\footnotetext{
${ }^{1}$ Si bien ha habido una prueba PISA más reciente con resultados disponibles, del 2012, la del 2009 enfatizó en lectura y por tanto es la última que nos permite el análisis aquí planteado, que incluye comparaciones con la prueba PISA 2000. ${ }^{2}$ Ver http://www.nichd.nih.gov/publications/pubs/nrp/pages/smallbook.aspx
} 
se lograrían hacia el inicio de la escolaridad, mientras que los dos últimos se pueden seguir desarrollando a lo largo de la vida.

En los Estados Unidos, Alvermann y colaboradores (2006) han hecho un balance de la enseñanza y el aprendizaje de la lectura. En cuanto al aprendizaje, durante los primeros cinco grados de escolaridad encuentran que los profesores más eficaces utilizan una variedad de técnicas orientadas a favorecer diversos procesos cognitivos y de auto regulación en los estudiantes, junto con un componente afectivo orientado a generar actitudes positivas hacia la lectura. No pareciera que se puede decir que el enfoque tradicional, basado en la fonética, sea superior a otros (aunque hay alguna evidencia que un énfasis en el enfoque fonético podría ser preferible en los primeros grados o para estudiantes con dificultades para adquirir la lectura y en cambio persistir en este enfoque en grados superiores de la primaria no sería beneficioso); en todo caso hay una gran variedad de técnicas de enseñanza de la lectura, cuya revisión excede los propósitos del presente artículo.

El aprendizaje de la lectura durante la secundaria por parte de jóvenes es un tema que ha sido menos estudiado que la adquisición de la lectura. La revisión de Alvermann y colaboradores (2006) del tema para los Estados Unidos, encuentra que la lectura se vio beneficiada cuando los docentes explícitamente enseñaron estrategias que requirieron que los estudiantes organicen, integren y reflexionen sobre textos PISA o informativos. Esto es lo que se podría llamar estrategias metacognitivas, que PISA incluye en sus cuestionarios como se verá más adelante. Alvermann y colaboradores también recomiendan incorporar en lo posible las necesidades e intereses de los estudiantes para planificar las sesiones de lectura. Así, sería deseable conectar lo que los estudiantes saben con lo que se pretende que aprendan, buscando desarrollar un sentimiento de logro y auto eficacia. Por ejemplo, Rodrígues-Valls (2010) identificó las expectativas en lectura y gustos literarios de estudiantes de secundaria en California, Estados Unidos. En entrevistas grupales e individuales con 50 estudiantes, los adolescentes criticaron la enseñanza de la lectura centrada en identificar información de los textos, dejando de lado el análisis crítico de los personajes o la trama del libro. Además, indicaron que los libros obligatorios de su plan de lectura no se conectaban con sus vidas ni intereses. El autor sugiere que sería necesario incluir la voz de los estudiantes en los diseños de los programas de lectura en la educación secundaria, con el fin de favorecer los procesos de lectura crítica.

En Finlandia se realizó un estudio por Linnakylä y Välijärvi (2006) que describe los principales factores que influyen en los altos resultados obtenidos por este país en las evaluaciones de PISA. Se encontró una pequeña brecha entre los estudiantes con un mayor y menor rendimiento (v.gr. tienen una de las menores desviaciones típicas entre los países evaluados). El nivel socioeconómico de los padres, el lugar de residencia en el que viven los jóvenes y el centro de estudios al que asisten, a diferencia de la mayoría de países, no son factores que influyen de manera determinante sobre el rendimiento de los alumnos. El alto rendimiento en Lectura se explica por la interrelación de diferentes factores: el compromiso con la lectura (explica un $22 \%$ de la varianza), el interés por la lectura $(18 \%)$, la comunicación cultural $(6 \%)$, los productos culturales a su disposición en el hogar (6\%) y el estatus socioeconómico de los padres (6\%). Por todo lo descrito, Linnakylä y Välijärvi (2006) sugieren que se trata de un sistema educativo de alta calidad, caracterizado por la equidad de oportunidades para todos los alumnos e influenciado por la homogeneidad cultural finlandesa. 
Por otro lado, Schubert y Becker (2010) estudian los factores asociados al rendimiento en lectura de los estudiantes alemanes medidos en las pruebas de PISA 2000 y PIRLS 2001. Encuentran que Alemania no obtuvo buenos resultados en la evaluación PISA 2000, en comparación con otros países de similares niveles de desarrollo, y además se observó una gran brecha en comprensión lectora asociada a inequidades sociales, desde el fin de la primaria. Estas diferencias se explican principalmente por los recursos socioeconómicos, el potencial de estimulación intelectual del hogar (definido por la cantidad de libros y presencia de periódicos) y las condiciones de aprendizaje al inicio de la escolaridad (por ejemplo, clima escolar). Hacia los 15 años de edad también se hallaron inequidades en el rendimiento lector por factores similares: prácticas culturales en el hogar (por ejemplo, visitas a eventos culturales), rendimiento hacia el final de la primaria, el contexto de enseñanza-aprendizaje, y la selectividad social en las transiciones educativas que se dan en el sistema educativo alemán. En otro estudio más reciente (Schwabe, McElvany y Trendtel, 2014) también en Alemania, se observó un mayor rendimiento lector por parte de los alumnos de 15 años de edad, que se describían altamente motivados hacia la lectura. Si bien se encontró que las niñas mostraron mejores resultados y niveles más altos de motivación intrínseca hacia la lectura, esto último no explicó su ventaja.

En México, el estudio de Hernández y Bazán (2016) analizó los datos de PISA 2009 con el fin de identificar las variables individuales y de contexto que explicaban en el rendimiento en lectura. Se definió el nivel socioeconómico del estudiante a partir del más alto nivel laboral y de estudios de los padres, y las posesiones en el hogar. Usando este indicador como base, se calculó la variable de contexto de la escuela como el promedio del nivel socioeconómico de los estudiantes de una misma escuela. Usando modelos jerárquicos multilineales, los hallazgos sugieren que el contexto de la escuela tiene un mayor impacto en el rendimiento en lectura, en comparación con algunas variables individuales. Sin embargo, este impacto varía según la heterogeneidad en la composición del contexto de la escuela y el tipo de gestión (privada o pública). A nivel individual, la variable más significativa fue la reprobación de la primaria, la cual afectó negativamente el rendimiento en lectura.

En Perú, Caro y otros (2004) analizaron los datos de PISA 2001 con el fin de identificar las asociación entre variables individuales y escolares con la comprensión lectora de los estudiantes. A nivel individual, los autores encuentran que la educación de los padres, las posesiones en el hogar, el grado en el que se encuentra el estudiante, la participación de los estudiantes en la escuela, su sentido de pertenencia y habitos de lectura están asociados de manera positiva y significativa con el rendimiento en lectura, mientras que el número de hermanos se asocia negativamente. A nivel de la escuela, contar con un docente con titulo profesional, un clima positivo en la escuela, la composición socioeconómica del alumnado y la gestión privada están asociadas de manera positiva y significativa con el rendimiento en lectura.

Benavides, León y Etesse (2014) analizaron las bases de datos de PISA 2000 y 2009 con la finalidad de estimar el impacto diferenciado del nivel socioeconómico de los estudiantes y sus familias en el rendimiento en lectura en America Latina. Ellos encontraron para el caso peruano que a pesar de la mejora en el rendimiento de los estudiantes, la brecha asociada a diferencias socioeconómicas se incrementó entre el 2000 y 2009. Asi mismo, los autores encontraron que las variables contextuales explicaban en mayor medida el rendimiento en lectura de los estudiantes peruanos, 
siendo el nivel socioeconómico promedio de la escuela la variable que más explica el rendimiento entre estudiantes. Así, el conjunto de estudios mencionados sugieren un fuerte rol del nivel socioeconómico, aunque hay pocos estudios específicamente sobre variedades vinculadas a oportunidades, estrategias y rendimiento en lectura en secundaria. Para entender lo que se pretende que aprendan los estudiantes peruanos, en la siguiente sección describimos brevemente el currículo peruano.

\subsection{El currículo peruano}

El 2009 en el Perú, el Diseño Curricular Nacional (DCN) marcaba lo que los docentes y estudiantes debían trabajar en las aulas. La comprensión de lectura está involucrada en mayor o menor medida en todas las áreas curriculares, pero en particular se vincula al área de comunicación. El DCN para secundaria (MED, 2008a) en comunicación buscaba en general que los estudiantes logren "comprender y producir textos diversos, en distintas situaciones comunicativas y con diferentes interlocutores" (p. 341). En comunicación, el DCN incluía tres organizadores: expresión y comprensión oral, comprensión de textos y producción de textos. En comprensión de textos se buscaba que los estudiantes logren "otorgar sentido a un texto a partir de las experiencias previas del lector y su relación con el contexto. Este proceso incluye estrategias para identificar la información relevante, hacer inferencias, obtener conclusiones, enjuiciar la posición de los demás y reflexionar sobre el proceso mismo de comprensión, con la finalidad de auto regularlo" (p. 342). Se incluía también un componente actitudinal de gusto por la lectura, y otro de reflexión crítica sobre la misma.

En otro texto del Ministerio se presentan algunas pautas para el desarrollo de capacidades comunicativas de los estudiantes (MED, 2006). Ahí se plantea que la comprensión de textos requiere los siguientes procesos: obtener información del texto a partir de la forma en que se presenta; obtener información del texto a partir del propósito para el que fue escrito; entender de manera general el contenido del texto; obtener información específica del texto; identificar y comprender los matices y sutilezas del lenguaje empleados por el escritor; y elaborar una interpretación del contenido del texto. Vista así, la comprensión de lectura aparece como una habilidad compleja que se puede desarrollar constantemente.

En el Perú para fomentar la lectura el Ministerio de Educación desde el 2006 ha desarrollado un plan lector's. Con ello se busca desarrollar hábitos de lectura y capacidades en comprensión lectora. Los textos a leer pueden ser de diferentes tipos, ya sea continuos (por ejemplo narrativos, argumentativos, descriptivos, expositivos y prescriptivos) o discontinuos (por ejemplo cuadros o gráficos). Así, con base en la normativa vigente, cada institución educativa debería encomendar a un equipo encargado de plantear su propio plan lector y supervisar su ejecución; se espera que en secundaria los estudiantes lean 12 libros al año, elegidos de acuerdo a sus características e intereses. Para facilitar la ejecución del plan lector, el Ministerio de Educación ha venido repartiendo módulos de biblioteca.

Cuánto de lo anterior efectivamente se cumple en las aulas y cuánto queda en planes, y por qué, son preguntas relevantes de política que no pueden ser contestadas plenamente

\footnotetext{
${ }^{3}$ El material incluido aquí ha sido adaptado de

http://www.oei.es/pdfs/orientaciones_especificas_plan_lector_SECUNDARIA.pdf
} 
con la información existente. Sin embargo en la sección de resultados se presentan algunos datos al respecto.

\subsection{Las evaluaciones PISA}

PISA es una evaluación organizada por la OCDE para evaluar a estudiantes de 15 años en comprensión de lectura, matemática y ciencias. Las tres áreas se evalúan cada tres años, pero en cada ronda el énfasis está puesto en una de ellas. El Perú participó en el primer PISA el 2000", y luego nuevamente el 2009; en ambos casos el énfasis estuvo en comprensión de lectura. El propósito general de PISA es evaluar en qué medida los jóvenes que están hacia el final de su educación básica han logrado adquirir conocimientos y habilidades consideradas esenciales para una participación plena en sociedades modernas (OCDE, 2010).

Para PISA el concepto de literacy (equivalente a alfabetización) es central, y se refiere a la "capacidad de los estudiantes de aplicar conocimientos y habilidades en áreas curriculares clave y analizar, razonar y comunicarse de manera efectiva cuando plantean, interpretan y solucionan problemas en una variedad de situaciones" (OCDE, 2010, p. 18; traducción de los autores). Específicamente para el caso de lectura, se definió alfabetización en lectura como "comprender, usar, reflexionar sobre e involucrarse con textos escritos, para lograr las metas personales, desarrollar el propio conocimiento y potencial, y participar en la sociedad" (OCDE, 2009, p. 23; traducción de los autores). Si bien los términos utilizados por PISA son diferentes de los planteados en el DCN, mencionados antes, en términos generales se podría decir que hay congruencia, aunque PISA no es una evaluación diseñada para evaluar currículos escolares. Sin embargo sería de esperar que el rendimiento en lectura y las otras dos áreas mencionadas debieran asociarse positivamente al grado de escolaridad de los estudiantes.

\section{Objetivos}

Siguiendo lo señalado al inicio del presente artículo, nos interesa explorar en primer lugar si las oportunidades de aprendizaje de los estudiantes se distribuyen de una manera equitativa entre diferentes grupos de nivel socioeconómico o en cambio reflejan disparidades similares a las observadas en el rendimiento. Las oportunidades de aprendizaje están definidas en cuanto a las características de las instituciones educativas a las que asisten los estudiantes de diferentes grupos, así como la disponibilidad de un ambiente farvorable en sus hogares; las oportunidades educativas en el presente estudio abarcan también las estrategias pedagógicas que implementan los docentes en las aulas y las estrategias de lectura reportadas por los estudiantes (estas presumiblemente influenciadas por el trabajo de los docentes). En segundo lugar, en el presente documento exploramos cambios en los patrones de rendimiento de diferentes grupos socioeconómicos (PISA del 2000, administrada en el Perú el 2001, y la del 2009). Finalmente, en el presente estudio exploramos el efecto principal y las interacciones entre nivel socioeconómico y oportunidades educativas sobre el rendimiento en lectura.

${ }^{4}$ Como se dijo antes, en el caso del Perú las pruebas se administraron el 2001. 


\section{Metodología}

\section{Muestra}

La muestra recogida en PISA 2000 y 2009 recoge datos de estudiantes en instituciones educativas seleccionadas aleatoriamente. De acuerdo al diseño muestral, es posible hacer inferencias a nivel nacional y por tipo de gestión educativa (público y privado), entre otras desagregaciones. En el Perú, los mejores rendimientos en pruebas previas se han encontrado para estudiantes que asisten a una institución educativa privada, urbana y para castellano hablantes. Dado que los estudiantes están agrupados en instituciones educativas, proponemos aquí una clasificación de estas que guiará el análisis. Esta se realizó clasificando las instituciones educativas de acuerdo al promedio socioeconómico de los estudiantes que asisten a ellas. Así, se crearon tres grupos (terciles), cada uno con el mismo número de instituciones educativas, para el $2000^{5}$ y $2009^{6}$. La justificación de presentar los resultados por terciles de instituciones educativas es analizar las oportunidades educativas y resultados que tienen estos grupos de estudiantes en ambos puntos del tiempo en el Perú. De acuerdo a criterios de equidad, no debería haber mayor diferencia en oportunidades ni resultados entre terciles; siguiendo el mismo principio, las brechas deberían mantenerse constantes o disminuir entre evaluaciones. A continuación se presentan las principales características demográficas de los estudiantes, así como de las instituciones educativas a las que asisten de acuerdo a lo recién definido.

Como se puede apreciar en el tabla 1, los estudiantes del tercil inferior es más probable que tengan como lengua materna una lengua indígena, tienen mayor retraso escolar, tienen menor probabilidad de haber asistido a educación inicial y provienen de familias con padres y madres menos educados; no hay mayores diferencias en estructura familiar, género o edad de los estudiantes. La muestra evaluada el 2009 era de padres y madres ligeramente más educados, lo cual va en línea con la expansión educativa de los últimos años.

Por diseño del estudio, el índice socioeconómico es más bajo en el tercil inferior y más alto en el superior (el promedio se fijó en o el año 2000 y el 2009). Lo interesante de los resultados es que la distancia entre el promedio del tercil superior respecto del inferior ha aumentado; así en el 2000 la diferencia socioeconómica entre el tercil inferior y medio era 0,58 desviaciones estándar, mientras que para el 2009 la diferencia entre estos mismos grupos se incrementa a 0,69 desviaciones estándar. La diferencia entre el tercil superior e inferior pasa de 1,37 desviaciones estándar a 1,56 desviaciones estándar entre el 2000 y 2009. Esto estaría sugiriendo una mayor segmentación entre estudiantes agrupados en instituciones educativas.

Con respecto a las características de los centros educativos a los que asisten los estudiantes de la muestra, lo primero que se debe notar es que la muestra de instituciones y estudiantes el 2009 es mayor, lo que permitiría mayor precisión en las estimaciones ese año.

\footnotetext{
${ }^{5}$ Para el nivel socioeconómico, en el caso del 2000 se cuenta en la base de datos de la OECD con un indicador que no es en estricto comparable con el índice calculado por esta misma institución el año 2009. Por tal motivo, se procedió a calcular un indicador comparable al del 2009, que incluye los diferentes aspectos socioeconómicos considerados en dicho año.

${ }^{6}$ Para el cálculo de los terciles, se procedió a calcular el promedio ponderado en lectura para cada una de las instituciones educativas para las evaluaciones del 2000 y 2009 para luego clasificar a las instituciones en los grupos alto, medio y bajo nivel socioeconómico.
} 
Tabla 1. Características de los estudiantes y las instituciones educativas evaluadas por terciles de nivel socioeconómico y año de evaluación

\begin{tabular}{|c|c|c|c|c|c|c|c|c|c|c|c|c|c|c|}
\hline & \multicolumn{7}{|c|}{2000} & \multicolumn{7}{|c|}{2009} \\
\hline & \multicolumn{2}{|c|}{$\begin{array}{c}\text { Inferior } \\
(\mathrm{n}=1192)\end{array}$} & \multicolumn{2}{|c|}{$\begin{array}{c}\text { Medio } \\
(\mathrm{n}=1700)\end{array}$} & \multicolumn{2}{|c|}{$\begin{array}{l}\text { Superior } \\
(\mathrm{n}=1537)\end{array}$} & \multirow[t]{2}{*}{$\begin{array}{l}\text { Nacional } \\
(\mathrm{n}=4429)\end{array}$} & \multicolumn{2}{|c|}{$\begin{array}{c}\text { Inferior } \\
(\mathrm{n}=1572)\end{array}$} & \multicolumn{2}{|c|}{$\begin{array}{c}\text { Medio } \\
(\mathrm{n}=2130)\end{array}$} & \multicolumn{2}{|c|}{$\begin{array}{l}\text { Superior } \\
(\mathrm{n}=2267)\end{array}$} & \multirow[t]{2}{*}{$\begin{array}{l}\text { Nacional } \\
(\mathrm{n}=5985)\end{array}$} \\
\hline Caracteristícas de los estudiantes & & & & & & & & & & & & & & \\
\hline Mujeres (\%) & 48.5 & a & 49.5 & a & 51.8 & a & 49.9 & 45.2 & a & 49.6 & $\mathrm{a}, \mathrm{b}$ & 52.6 & $\mathrm{~b}$ & 49.5 \\
\hline Edad promedio & 15.7 & a & 15.7 & a & 15.7 & a & 15.7 & 15.8 & a & 15.8 & a & 15.8 & a & 15.8 \\
\hline Castellano hablantes en casa (\%) & 87.4 & a & 97.2 & $\mathrm{~b}$ & 99.2 & c & 94.7 & 85.0 & a & 98.0 & $\mathrm{~b}$ & 99.0 & $\mathrm{c}$ & 94.7 \\
\hline En el grado para su edad (\%) & 35.1 & a & 48.9 & $\mathrm{~b}$ & 77.8 & $\mathrm{c}$ & 53.9 & 47.4 & a & 70.5 & $\mathrm{~b}$ & 89.1 & $\mathrm{c}$ & 71.3 \\
\hline Asistió a educación inicial (\%) & - & & - & & - & & - & 74.6 & a & 84.2 & $\mathrm{~b}$ & 92.3 & $\mathrm{c}$ & 84.9 \\
\hline \multicolumn{15}{|l|}{ Estructura familiar (\%) } \\
\hline Familia nuclear & 71.1 & a & 66.9 & a & 70.6 & a & 69.3 & 79.4 & a & 76.6 & a & 72.4 & $\mathrm{~b}$ & 75.8 \\
\hline Padre/madre soltera & 17.0 & a & 20.8 & $\mathrm{~b}$ & 19.7 & $\mathrm{a}, \mathrm{b}$ & 19.3 & 16.1 & a & 18.4 & a & 23.6 & $\mathrm{~b}$ & 19.7 \\
\hline Otro & 11.9 & $a, b$ & 12.3 & a & 9.7 & $\mathrm{~b}$ & 11.4 & 4.5 & a & 5.0 & a & 4.0 & a & 4.5 \\
\hline Padre con educación universitaria o más (\%) & 11.5 & a & 22.9 & $\mathrm{~b}$ & 48.5 & c & 27.6 & 13.0 & a & 24.5 & $\mathrm{~b}$ & 50.3 & $\mathrm{c}$ & 31.3 \\
\hline Madre con educación universitaria o más (\%) & 4.0 & a & 12.8 & $\mathrm{~b}$ & 36.0 & c & 17.6 & 6.9 & a & 13.1 & $\mathrm{~b}$ & 33.1 & $\mathrm{c}$ & 19.1 \\
\hline Nivel Socioeconomico (indice) & -0.7 & a & -0.1 & $\mathrm{~b}$ & 0.7 & c & 0.0 & -0.8 & a & -0.1 & $\mathrm{~b}$ & 0.7 & $\mathrm{c}$ & 0.0 \\
\hline \multicolumn{15}{|l|}{ Caracteristicas de las institutciones educativas } \\
\hline Numero de institución educativas & 59.0 & & 59.0 & & 59.0 & & 177.0 & 80.0 & & 80.0 & & 80.0 & & 240.0 \\
\hline Promedio de estudiantes por clase & 21.9 & a & 33.0 & $\mathrm{~b}$ & 23.9 & $\mathrm{~b}$ & 24.5 & 18.0 & a & 21.6 & $\mathrm{~b}$ & 22.6 & $\mathrm{~b}$ & 20.1 \\
\hline Alumnos por docente & 18.1 & a & 23.5 & $\mathrm{~b}$ & 15.8 & $\mathrm{a}, \mathrm{c}$ & 18.3 & 12.9 & a & 11.5 & a & 11.2 & a & 12.1 \\
\hline Alumnos por computadora & 282.6 & a & 203.5 & $\mathrm{~b}$ & 37.4 & $\mathrm{c}$ & 66.8 & 52.7 & a & 35.5 & $\mathrm{~b}$ & 19.6 & $\mathrm{c}$ & 29.2 \\
\hline Horas de clase semanales en comunicación & - & & - & & - & & - & 4.9 & $\mathrm{a}, \mathrm{b}$ & 5.1 & a & 4.7 & $\mathrm{~b}$ & 4.9 \\
\hline Horas de clase semanales en matemática & - & & - & & - & & - & 4.8 & a & 5.1 & $\mathrm{~b}$ & 5.6 & $\mathrm{c}$ & 5.2 \\
\hline Horas de clase semanales en ciencias & - & & - & & - & & - & 3.4 & a & 3.7 & $\mathrm{~b}$ & 4.0 & $\mathrm{c}$ & 3.8 \\
\hline
\end{tabular}

Notas: Todos los cuadros y gráficos del presente documento han sido elaborados por los autores. Indicadores con distinto superíndice en una misma fila el mismo año indican que la diferencia entre grupos es estadísticamente significativa $(\mathrm{p}<0,05)$ de acuerdo al t-test para muestras independientes ( $\mathrm{v}$. gr. a es diferente de b y b es diferente de c). Para el cálculo de las diferencias por grupo fue usado el diseño muestral de la encuesta. No se muestran las horas de clase para el 2000 debido a que la pregunta en ese año no era comparable con la del 2009. Las horas de clase reportadas son cronológicas (60 minutos).

Fuente: Elaboración propia a partir de PISA 2000 y 2009. 
En cuanto a las diferencias entre grupos, el número de estudiantes por institución educativa se redujo entre el 2000 y 2009, aunque en ambos casos las más grandes son las del tercil medio. Esto se explica porque se trata de instituciones educativas urbanas, versus las del grupo inferior que son en gran parte rurales y las del superior que son en muchos casos privadas. El número de computadoras disponibles por institución educativa haa aumentado, aunque se nota claramente una gradiente que corresponde con el nivel socioeconómico de las instituciones educativas. Este es un primer indicador de inequidad en la provisión de insumos educativos (v. gr. los estudiantes de los tres grupos deberían tener igual acceso a computadoras y otros insumos, o incluso en una lógica de priorización podrían tener más computadoras). También de manera notable, el número de estudiantes por docente se ha reducido en la segunda evaluación. Esto podría tener implicancias para los resultados, pues un docente con menos estudiantes tendría más tiempo para trabajar con cada individuo (aunque el efecto en el análisis del año 2009 no fue significativo en la evaluación del 2009; los resultados se presentan en el Cuadro 6). Finalmente, en cuanto a las horas de clase por área (reportadas por los mismos estudiantes), se tiene una gradiente asociada al nivel socioeconómico en matemática y ciencias, más no en comunicación (que es el área que se vincula directamente con el aprendizaje de la lectura); en esta área los estudiantes de nivel socioeconómico alto reportan menos horas semanales de comunicación.

Índices de contexto en los cuestionarios de PISA

En todas sus evaluaciones, PISA ha administrado instrumentos de contexto a los directores de las instituciones educativas y estudiantes participantes. El cuestionario a directores del 2009 cubrió los siguientes temas: estructura y organización de la institución educativa, características del cuerpo docente y de los estudiantes, recursos disponibles en la institución educativa, currículo y evaluación, clima escolar prácticas y políticas en la institución educativa, y características del director.

El cuestionario a los estudiantes del 2009 incluyó preguntas sobre su historia educativa, involucramiento en prácticas de lectura, tiempo de instrucción, aprendizaje y evaluación, clima del aula y la institución educativa, y disponibilidad y uso de bibliotecas. Algunos de estos temas fueron también incluidos en los cuestionarios del 2000. La definición y justificación de cada constructo utilizado aparece en el marco conceptual de la evaluación (OCDE, 2009).

El uso de estos índices y la clasificación de las instituciones educativas por terciles socioeconómicos, permiten analizar la equidad del sistema educativo peruano a través del tiempo. Así, estos análisis nos permiten ver cómo ha cambiado la brecha entre los tres grupos de institución educativa mencionados antes entre el 2000 y el 2009. En una sociedad con un sistema educativo eficaz se esperaría que los promedios en habilidades aumenten con el tiempo, y en una sociedad justa se esperaría que las brechas entre grupos de estudiantes de diferente nivel socioeconómico tiendan a disminuir y eventualmente desaparecer. En la siguiente sección se presentan los principales resultados relacionados con la disponibilidad de textos de lectura, la lectura como práctica de los estudiantes y las estrategias pedagógicas de los docentes para promoverla. Las definiciones y descripciones de cada uno de los índices utilizados en el análisis se encuentran en el Anexo 1. 


\section{Resultados}

\subsection{Disponibilidad de textos, hábitos, estrategia y pedagogía de la Lectura}

Un primer tema a analizar es la disponibilidad de textos para leer. La figura 1 muestra el porcentaje de estudiantes que cuenta con 11 libros o más en el hogar. Se encuentran diferencias estadísticamente significativas entre los terciles de nivel socioeconómico; así los estudiantes del tercil superior reportan en un porcentaje significativamente mayor tener más de 10 libros en el hogar, en comparación al tercil medio e inferior. Como se puede apreciar, la disponibilidad de libros se ha incrementado en los tres terciles.

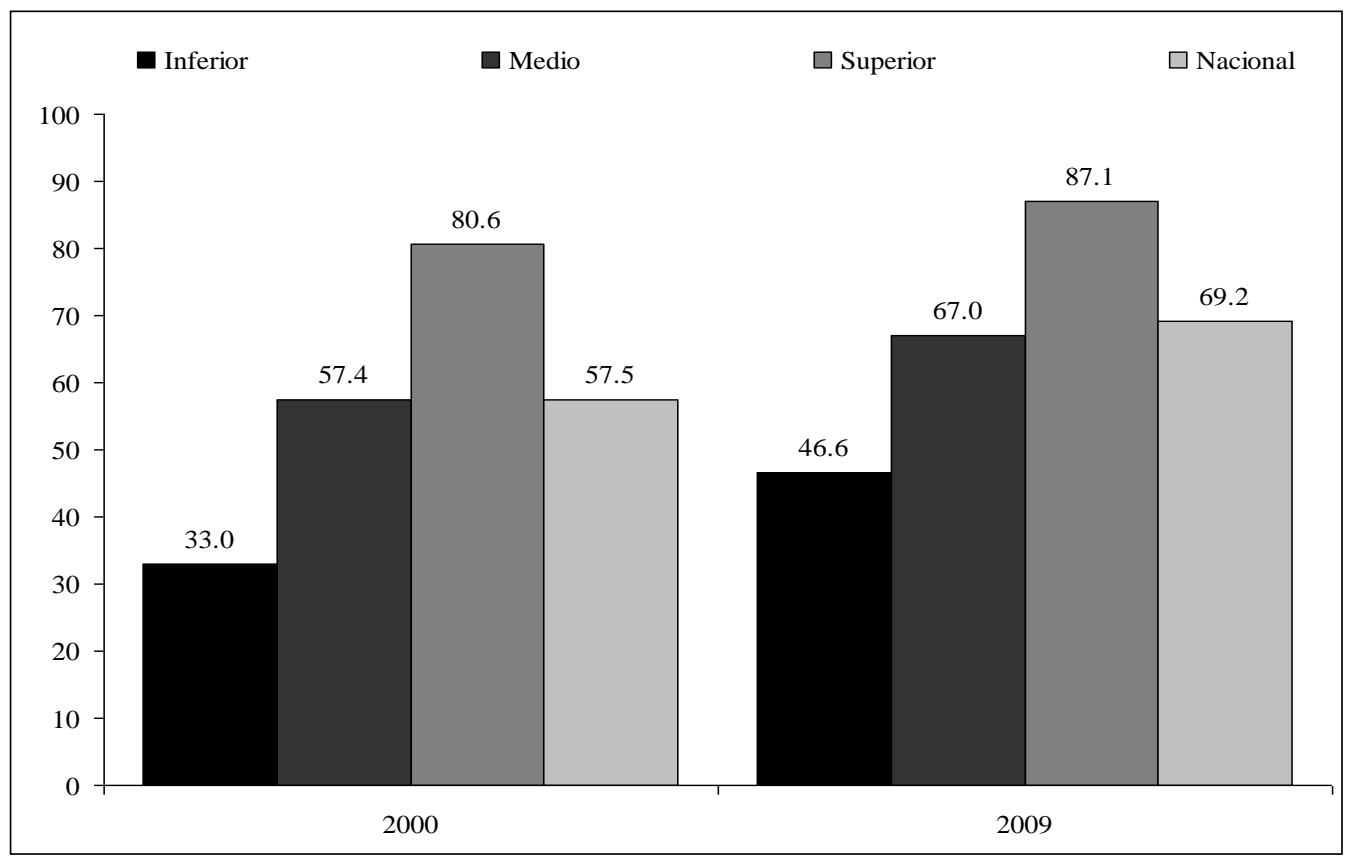

Figura 1. Estudiantes con 11 libros o más en el hogar, por terciles de nivel socioeconómico y año de aplicación (porcentaje)

Nota: El cálculo de los porcentajes se hizo sobre la base del diseño muestral.

Fuente: Elaboración propia a partir de PISA 2000 y 2009.

La literatura resalta la importancia de la motivación para leer. Al respecto, uno de los temas incluidos en el cuestionario es si los estudiantes leen por placer, indagando de ser el caso cuáles eran los materiales más usados en este espacio (ver tabla 2). En el índice generado con los ítems se puede apreciar que los estudiantes del tercil inferior muestran mayor interés y motivación por la lectura ${ }^{7}$. Entre los principales materiales usados para la lectura por placer se reporta en primer lugar los periódicos $(74 \%$ el 2009 para el promedio nacional), seguido de textos de ficción (56\%), libros de historietas y revistas (ambos con $48 \%$ ) y de no ficción (40\%).

\footnotetext{
${ }^{7}$ No se presentan aquí datos descriptivos sobre cada uno de los ítems para cada una de las escalas por motivo de espacio, pero se puede solicitar la información a los autores.
} 
Tabla 2. Placer por la Lectura (promedio simple estandarizado y error estándar)

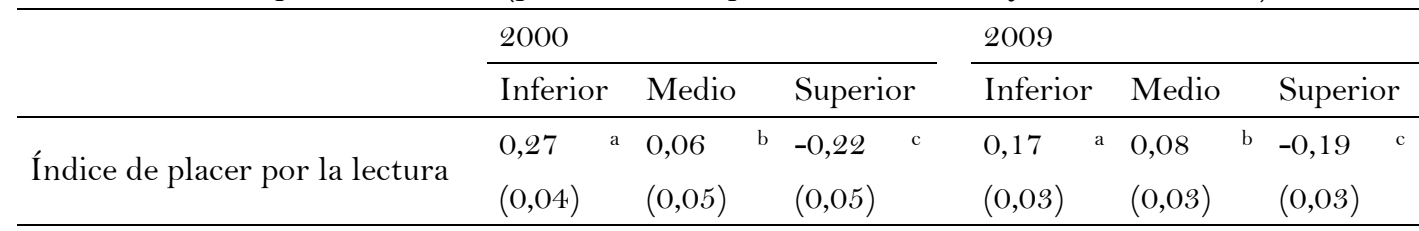

Nota: Promedios con distintos superíndices indican que las diferencias entre grupos son estadísticamente significativas al 5\% de acuerdo al t-test para muestras independientes. Para el cálculo de los porcentajes y errores estándar se usó el diseño muestral. El promedio se fijó en o.

Fuente: PISA 2000 y 2009.

\subsection{Estrategias metacognitivas en la comprensión lectora en los estudiantes peruanos}

El 2009, PISA incluyó preguntas sobre estrategias metacognitivas, que se definen como el conocimiento y habilidad para usar una variedad de procedimientos para procesar textos. La investigación sobre el tema sugiere que este tipo de estrategias está asociado a mejores rendimientos. En el cuestionario se indagó por estrategias de memorización, control, elaboración, recuperación y síntesis durante la lectura; los resultados para estas se reportan en la tabla 3.

Tabla 3. Índices sobre estrategias metacognitivas en la comprensión lectora, por terciles de nivel socioeconómico 2009 (promedio simple estandarizado y error estándar)

\begin{tabular}{|c|c|c|c|c|c|}
\hline & \multicolumn{5}{|c|}{2009} \\
\hline & Inferior & r Medic & & Superio & \\
\hline Índice de uso de estrategias de memorización & $\begin{array}{l}0,12{ }^{\mathrm{a}} \\
(0,04)\end{array}$ & $\begin{array}{cc}0,08 \\
& (0,03)\end{array}$ & $\mathrm{a}$ & $\begin{array}{l}-0,16 \\
(0,03)\end{array}$ & $\mathrm{b}$ \\
\hline Índice de uso de estrategias de elaboración & $\begin{array}{c}0,01 \quad \mathrm{a} \\
(0,04)\end{array}$ & $\begin{array}{ll}0,08 \\
& (0,03)\end{array}$ & $\mathrm{a}$ & $\begin{array}{l}-0,12 \\
(0,03)\end{array}$ & b \\
\hline Índice de uso de estrategias de control & $\begin{array}{l}-0,07 \quad a \\
(0,04)\end{array}$ & $\begin{array}{cc}0,04 \\
(0,03)\end{array}$ & b & $\begin{array}{l}-0,03 \\
(0,03)\end{array}$ & a,b \\
\hline $\begin{array}{l}\text { Índice de uso de estrategias de compresión y recuperación de } \\
\text { información }\end{array}$ & $\begin{array}{l}-0,21 \quad a \\
(0,04)\end{array}$ & $\begin{array}{l}-0,04 \\
\\
(0,02)\end{array}$ & b & $\begin{array}{l}0,14 \\
(0,04)\end{array}$ & c \\
\hline Índice de uso de estrategias de síntesis & $\begin{array}{l}-0,28 \quad a \\
(0,03)\end{array}$ & $\begin{array}{ll}-0,04 \\
& (0,02)\end{array}$ & b & $\begin{array}{l}0,19 \\
(0,04)\end{array}$ & c \\
\hline
\end{tabular}

Nota: Promedios con distintos superíndices indican que las diferencias entre grupos son estadísticamente significativas al $5 \%$ de acuerdo al t-test para muestras independientes. Para el cálculo de los porcentajes y errores estándar se usó el diseño muestral. El promedio para cada índice se fijó en 0 .

Fuente: Elaboración propia a partir de PISA 2000 y 2009.

En el índice de estrategias de memorización, el tercil superior es estadísticamente más bajo que los otros dos grupos. En el índice de uso de estrategias de elaboración y estrategias de control no existe un patrón claro entre los terciles. El cuarto índice alude a la implementación de estrategias metacognitivas durante la lectura, orientadas a guardar o evocar diferentes aspectos de lo leído. Se aprecia que son los estudiantes del tercil superior los que en mayor medida hacen uso de este tipo de estrategias, a diferencia del tercil medio e inferior.

Finalmente, se preguntó a los estudiantes sobre el uso de estrategias relacionadas con la síntesis de la información leída. Los resultados muestran que los estudiantes del tercil superior reportan utilizar con mayor frecuencia estrategias que tienen que ver con procesar activamente la información (por ejemplo escribir el resumen y revisarlo para 
verificar que contenga las ideas principales), mientras que en el estrato medio e inferior predominan estrategias más mecánicas (como por ejemplo copiar con exactitud las oraciones).

\subsection{Las prácticas pedagógicas hacia Lectura en las instituciones educativas}

PISA también incluye una serie de preguntas vinculadas a las prácticas pedagógicas y vínculos entre estudiantes, resultados que se reportan en la tabla 4. En la tabla 4, se puede apreciar que no existe un patrón claro en cuanto a la percepción del uso de estrategias de estimulación docente (p.ej.: docente motiva a los estudiantes a expresar sus opiniones) y estructuración (p.ej.: preguntar si entendieron las tareas); solo se aprecian diferencias estadísticamente significativas entre los estudiantes del tercil superior y medio ${ }^{8}$.

En el 2000 como en el 2009, se indagó acerca de las relaciones entre docentes y alumnos, y la percepción de los estudiantes acerca del clima de disciplina en el aula. Se puede apreciar que no existen diferencias entre terciles socioeconómicos con respecto al índice de relaciones profesor-alumno. El tema de clima en el aula también fue incluido para los dos años de evaluación, apreciándose solo una diferencia significativa (los estudiantes del tercil inferior perciben un mejor clima del aula a comparación de los estudiantes del tercil medio) para el 2000 y ninguna diferencia entre terciles en el 2009 ninguna.

Finalmente, el 2009 PISA incluyó también una sección sobre actividades extracurriculares (p. ej.: actividades de voluntariado y deportes) ofrecidas por la institución educativa. Los resultados sugieren que estas actividades son más frecuentes en instituciones educativas del tercil socioeconómico superior, seguidas del medio y finalmente el inferior.

Tabla 4. Índices sobre prácticas pedagógicas hacia lectura, por terciles de nivel socioeconómico 2009 (promedio simple estandarizado y error estándar)

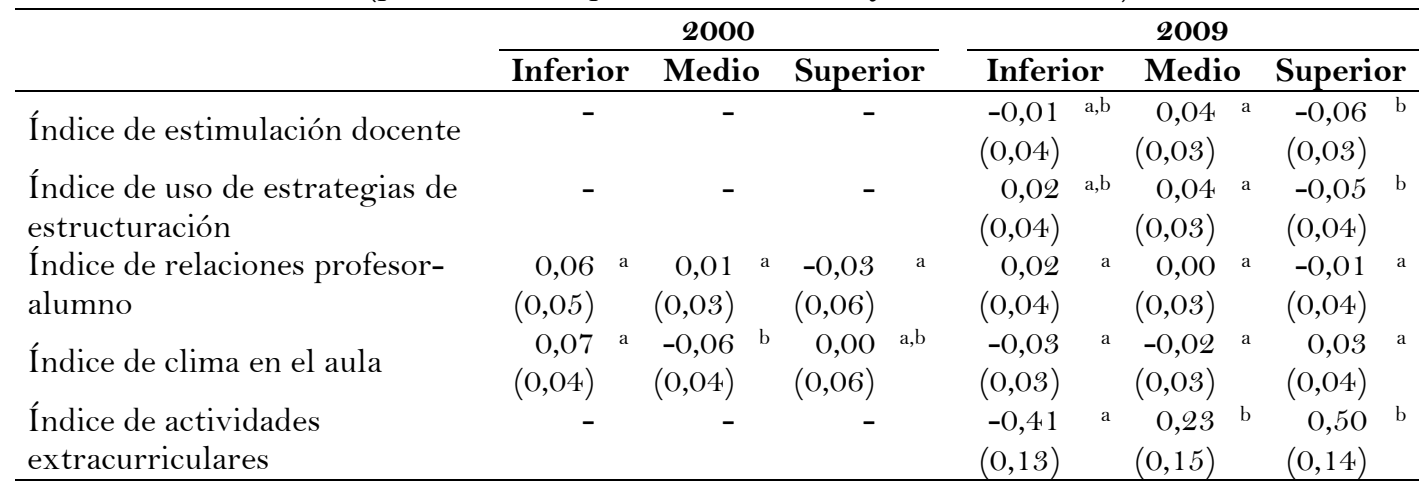

Nota: Promedios con distintos superíndices indican que las diferencias entre grupos son estadísticamente significativas al $5 \%$ de acuerdo al t-test para muestras independientes. Para el cálculo de los porcentajes y errores estándar se usó el diseño muestral. El promedio para cada índice se fijó en 0 .

Fuente: PISA 2000 y 2009.

\footnotetext{
${ }^{8}$ Solo se cuenta con información para el año 2009 para estos índices y el de actividades extracurriculares.
} 


\subsection{Puntajes de los estudiantes peruanos en PISA}

En PISA 2000 Perú resultó último en el ranking entre 43 países evaluados, pero se debe recordar que se trata de una evaluación donde participan principalmente países miembros de la OCDE (Perú no es miembro). Solo el 1,1\% de los estudiantes peruanos se ubicaron en los dos niveles superiores de rendimiento ( 4 y 5 ) y 79,6\% en los dos inferiores (o y 1). En la evaluación del 2009 Perú resultó en el puesto 63 entre 65 países, pero fue el país que más mejoró su promedio desde el 2000. Aun así el promedio de los estudiantes peruanos fue el más bajo entre los países latinoamericanos, junto a Panamá. Todos los países de la región se ubicaron significativamente por debajo del promedio de la OCDE (ver tabla 5).

Tabla 5. Puntajes promedio y diferencias en las pruebas de lectura entre 2000 y 2009 en Perú y otros países de Latinoamérica

\begin{tabular}{|c|c|c|c|c|c|}
\hline & \multicolumn{2}{|c|}{2000} & \multicolumn{2}{|c|}{2009} & \multirow{2}{*}{$\begin{array}{c}\text { DIFERENCIA } \\
\text { PROMEDIO }\end{array}$} \\
\hline & Promedio & EE & Promedio & $\mathbf{E E}$ & \\
\hline Argentina & 418,3 & $(9,9)$ & 398,3 & $(4,6)$ & $-20,0$ \\
\hline Brasil & 396,0 & $(3,1)$ & 411,8 & $(2,7)$ & 15,7 \\
\hline Chile $^{1}$ & 409,6 & $(3,6)$ & 449,4 & $(3,1)$ & 39,8 \\
\hline México $^{1}$ & 422,0 & $(3,3)$ & 425,3 & $(2,0)$ & 3,3 \\
\hline Perú & 327,1 & $(4,4)$ & 369,7 & $(3,9)$ & 42,6 \\
\hline OCDE promedio $23^{2}$ & 501,0 & $(0,7)$ & 499,0 & $(0,6)$ & $-2,0$ \\
\hline
\end{tabular}

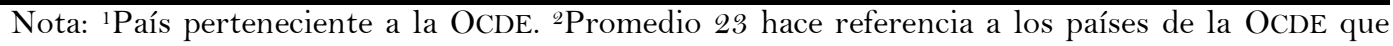
estuvieron presentes en todas las evaluaciones hechas por PISA. Para el cálculo de los errores estándar (EE) se usó el método de Balanced Repeated Replication (BRR).

Fuente: PISA 2000 y 2009.

En el presente estudio interesa conocer no solamente el promedio sino la inequidad a través del tiempo. La figura 2 presenta los promedios por terciles para 2000 y 2009. El tercil medio muestra el mayor crecimiento (44 puntos), seguido del tercil superior con $38 \mathrm{y}$ el inferior con 24 . En otras palabras, si bien la brecha entre el tercil medio y superior se habría cerrado ligeramente, la brecha entre ambos con el tercil inferior se habría incrementado.

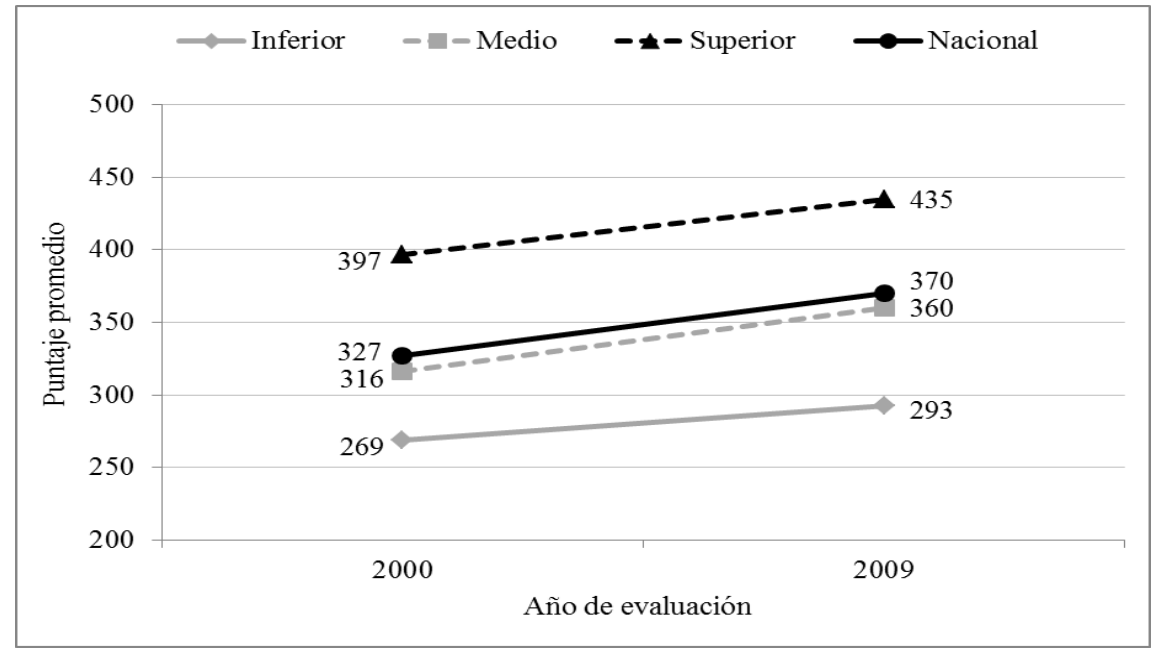

Figura 2. Puntaje promedios en la prueba de Comprensión lectora por terciles de nivel socioeconómico y año de aplicación

Fuente: Elaboración propia a partir de PISA2000 y 2009. 
La mejora en el rendimiento promedio para Perú se dio en todos los niveles. Por ejemplo, los estudiantes por debajo del nivel 2 (considerado mínimamente aceptable por PISA) disminuyó en 14,8\% entre el 2000 y el 2009, y los estudiantes en el nivel 5 aumentaron en $0,4 \%$ en el mismo período. De todos modos, $64,8 \%$ de los estudiantes peruanos se encontraron por debajo del nivel 2 el 2009. Sin embargo, el grupo de estudiantes del tercil socioeconómico superior es el que logra un mayor incremento en los niveles más altos de PISA, pasando de 3,4\% en el 2000 a 7,6\% el 2009. El tercil medio tuvo un incremento de 0.4 puntos porcentuales y el inferior no mostró incremento (ver figura 3).

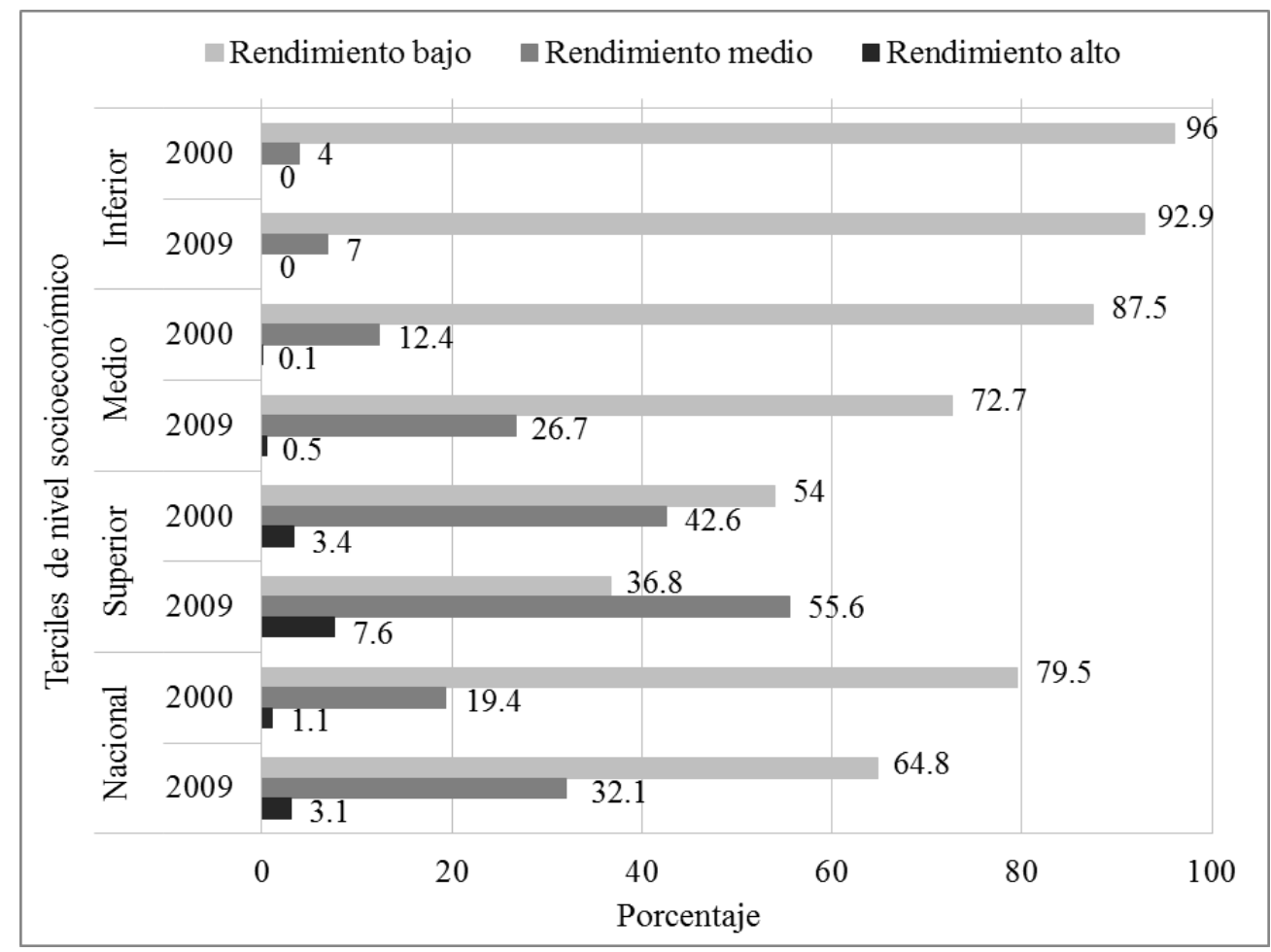

Figura 3. Niveles de desempeño en Comprensión lectora por terciles de nivel socioeconómico y año de aplicación

Nota: El nivel de rendimiento bajo comprende los niveles 0 y 1 , el nivel medio el 2 y 3 , mientras el alto los niveles 4 y 5 .

Fuente: Elaboración propia a partir de PISA2000 y 2009.

Si bien PISA presenta resultados de lectura en tres subescalas: obtención de información, integración e interpretación, y reflexión y evaluación, el análisis que se presenta a continuación se centra en el puntaje global en lectura (la posición relativa de Perú es similar en las tres subescalas).

\subsection{Factores asociados al rendimiento en comprensión lectora}

Las anteriores secciones han presentado prácticas auto reportadas de lectura y rendimiento. En la presente se analiza cuál es la asociación entre estas variables, para explorar luego implicancias de política. El análisis incluye variables a nivel del estudiante (individuales) y a nivel de la institución educativa. En los análisis de las bases de datos se encontró un alto porcentaje de valores faltantes a nivel de estudiante en lo 
relacionado con prácticas pedagógicas. A nivel de la institución educativa, el ratio de estudiantes por docente contaba con la mayor cantidad de valores faltantes. Ambos aspectos hacían que al final la muestra se redujera en cerca del $30 \%$, lo cual podía traer problemas en la estimación de las relaciones dado que se podría estar analizando una muestra sesgada. Es así que se realizó la imputación de valores faltantes en variables a nivel del individuo y de la institución educativa; ambos procesos se realizaron de manera separada. Para tal fin, se usó el método de imputación por cadena de ecuaciones (Imputation by Chained Equations). Este método planteado por Royston (2004) usa la función de densidad de cada variable pero condicionada a las otras variables usadas para la imputación. Las variables que se imputaron a nivel individual fueron lectura por placer, estrategias de memorización, estrategias de control, estrategias de comprensión y recuperación de información, estrategias de síntesis, estimulación docente y clima en el aula, mientras que a nivel de la institución educativa solo se imputó el ratio alumno por docente.

Los gráficos de distribución de las variables sin imputar e imputadas no afectaron la distribución de las variables. Para el analizar cuál es la asociación entre estas variables y rendimiento se utilizó un modelo jerárquico multinivel (Raudenbush y Bryk, 2002), que permite una mejor estimación de los errores estándar a nivel individual y de la institución educativa. Las principales variables de interés son las vinculadas a los índices referidos a prácticas en la institución educativa, que fueron mencionados antes. Las variables consideradas en el siguiente análisis y sus definiciones se incluyen en el Anexo 2. En cuanto a los índices, se seleccionaron aquellos con una relación significativa con la variable dependiente (ver análisis en Anexo 3). Se incluyeron además otras variables de control, de acuerdo a estudios previos (Cueto, 2007).

En el análisis se estimó el efecto principal de las variables de estrategias y prácticas pero también las interacciones entre estas y el nivel socioeconómico de la institución educativa. Los resultados se presentan en la tabla 6. El modelo 1 solo considera las variables del estudiante y su familia, el modelo 2 incluye variables a nivel de la IE, el modelo 3 incluye el nivel socioeconómico de la institución educativa, el modelo 4 incluye las variables pedagógicas a nivel del individuo y el modelo 5 incluye las interacciones de las variables pedagógicas con los terciles de nivel socioeconómico. A nivel del individuo se encontró que las mujeres tienen mayor rendimiento (aunque la diferencia deja de ser significativa en los modelos 4 y 5), igual que los que tienen castellano como lengua materna, los de mayor nivel socioeconómico y los que están en el grado correspondiente para su edad o están adelantados. A nivel de la institución educativa, se puede apreciar que las IE urbanas, privadas, con mayor número de estudiantes y de mayor tercil socioeconómico agregado tuvieron promedios más altos que sus pares. El número de estudiantes por docente no se relacionó con el rendimiento.

Como se dijo antes, el foco del análisis está en las variables pedagógicas, que se incluyen en los modelos 4 y 5 . Como se menciona en el anexo 1, todas las variables incluidas tuvieron una asociación significativa con rendimiento, sin embargo al incluirlas con las demás, las que continúan mostrando coeficientes significativos son placer por la lectura, memorización, control, comprensión, síntesis, relaciones profesor-alumno y clima en el aula; memorización tiene un coeficiente negativo y las demás coeficientes positivos. En cuanto a las interacciones de las variables pedagógicas con los terciles de nivel socioeconómico de las instituciones educativas, solo encontramos resultados 
estadísticamente significativos para clima en el aula. Por lo demás los efectos reportados en el modelo 4 son similares a través de los terciles.

La figura 4 muestra el tamaño del efecto de cada una de las variables pedagógicas incluidas en el modelo. Se puede apreciar que las estrategias de comprensión (E.S.: 0.09 DE), las estrategias de memorización (E.S.: -0.09 DE), el placer por la lectura (E.S.: 0.07 DE) y las estrategias de síntesis (E.S.: $0.07 \mathrm{DE}$ ) son aquellas que cuentan con un mayor tamaño del efecto. Si bien los tamaños del efecto son pequeños ${ }^{9}$, se debe tomar en consideración que estos efectos han sido estimados luego de controlar estadísticamente por las características de los estudiantes y de las instituciones educativas.

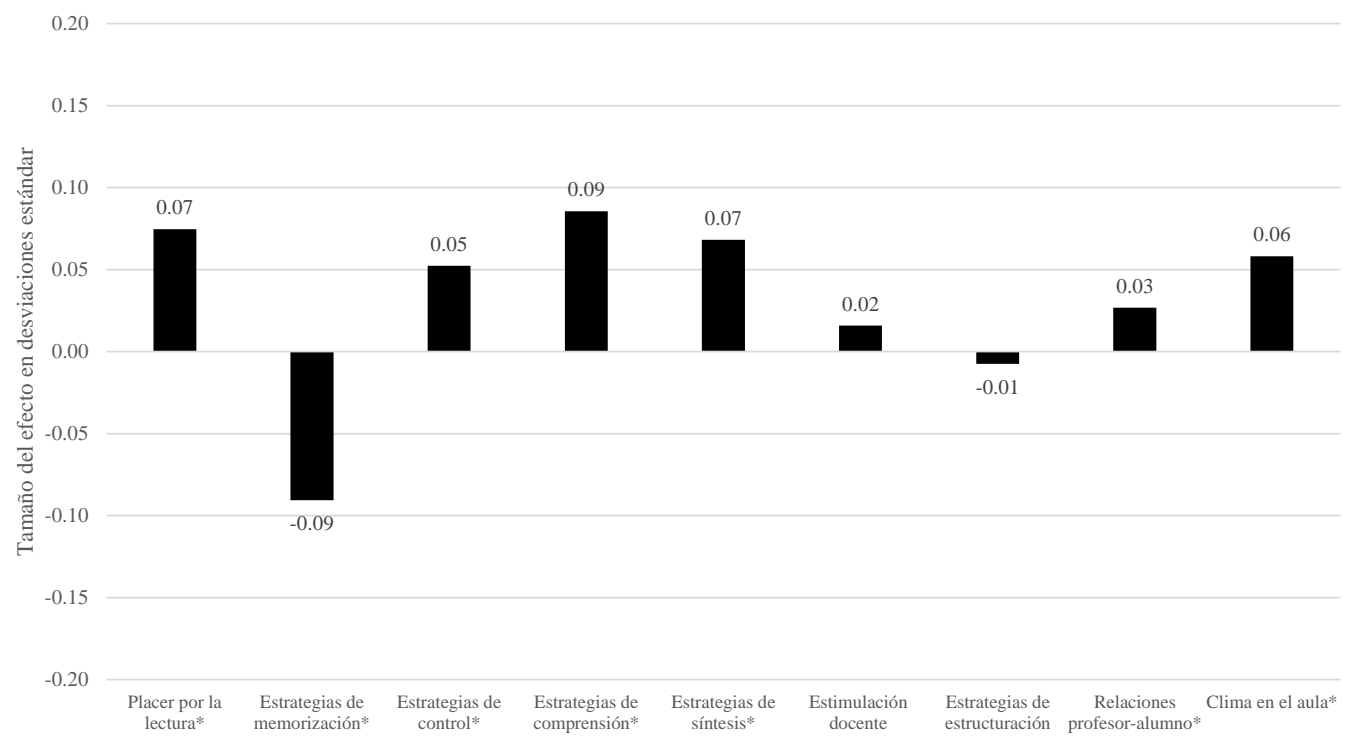

Figura 4. Tamaño del efecto de las variables pedagógicas en el análisis multivariado Nota: * Efectos estadísticamente significativos.

Fuente: Elaboración propia.

\section{Discusión e implicancias para políticas e investigación}

Como se mencionó al inicio del presente artículo, el presente análisis tiene un foco en equidad. Así, a lo largo del artículo se han presentado resultados para estudiantes agrupados en terciles por el nivel socioeconómico promedio de la institución educativa a la que asisten. Los resultados muestran que hay una segmentación clara del alumnado; los estudiantes del tercil inferior tienen en promedio padres menos educados, menos años de educación inicial y es más probable que tengan una lengua materna indígena. Lo opuesto se observa en el tercil superior, que además en mayor proporción asiste a instituciones educativas urbanas y privadas.

${ }_{9}^{9}$ De acuerdo a Cohen (1988) un tamaño del efecto pequeño es 0.20 DE, uno mediano es O.50 DE y uno grande es 0.80 DE, aunque los efectos observados en educación a menudo son menores que estos (McEwan, 2015). 
Tabla 6. Análisis Lineal Jerárquico para comprensión lectora

\begin{tabular}{|c|c|c|c|c|c|c|c|c|c|c|c|c|c|c|c|}
\hline \multirow{3}{*}{ A nivel del estudiante } & \multicolumn{3}{|c|}{$\begin{array}{l}\text { MODELO } 1 \\
\end{array}$} & \multicolumn{3}{|c|}{ MODELO 2} & \multicolumn{3}{|c|}{ MODELO 3} & \multicolumn{3}{|c|}{ MODELO 4 } & \multicolumn{3}{|c|}{ MODELO 5} \\
\hline & \multirow[t]{2}{*}{$\beta$} & \multicolumn{2}{|c|}{ ee $(\beta)$} & $\beta$ & \multicolumn{2}{|c|}{ ee $(\beta)$} & B & \multicolumn{2}{|l|}{ ee $(\beta)$} & $\beta$ & \multicolumn{2}{|l|}{ ee $(\beta)$} & $\beta$ & \multicolumn{2}{|c|}{ ee $(\beta)$} \\
\hline & & & & & & & & & & & & & & & \\
\hline Género (mujer) & 7,82 & $(2,29)$ & *** & 7,63 & $(2,28)$ & *** & 7.57 & $(2.25)$ & *** & 1.85 & $(2.34)$ & & 2.22 & $(2.33)$ & \\
\hline Lengua (español) & 24,62 & $(5,61)$ & **** & 23,38 & $(5,39)$ & **** & 22.90 & $(5.24)$ & **** & 20.50 & $(5.57)$ & *** & 19.29 & $(5.60)$ & *** \\
\hline Estructura familiar (familia nuclear) & $-4,43$ & $(2,33)$ & & $-4,14$ & $(2,35)$ & & -3.98 & $(2.37)$ & & -2.82 & $(2.32)$ & & -2.97 & $(2.32)$ & \\
\hline Estructura familiar (familia mixta) & $-7,77$ & $(5,42)$ & & $-8,42$ & $(5,45)$ & & -8.36 & $(5.43)$ & & -7.29 & $(5.23)$ & & -8.01 & $(5.22)$ & \\
\hline Nivel socioeconómico & 10,52 & $(1,13)$ & **** & 9,20 & $(1,11)$ & **** & 8.38 & $(1.13)$ & **** & 7.96 & $(1.01)$ & **** & 8.12 & $(1.02)$ & **** \\
\hline Asistió a educación inicial & 5,61 & $(2,75)$ & * & 5,50 & $(2,76)$ & & 4.98 & $(2.73)$ & & 4.31 & $(2.63)$ & & 4.53 & (2.66) & \\
\hline Grado para la edad & 28,88 & $(1,20)$ & **** & 28,47 & $(1,21)$ & ***** & 28.08 & $(1.21)$ & **** & 24.30 & $(1.20)$ & **** & 24.18 & $(1.22)$ & **** \\
\hline Placer por la lectura & & & & & & & & & & 7.27 & $(0.99)$ & **** & 3.87 & $(2.84)$ & \\
\hline Placer por la lectura * Nivel socioeconómico IE & & & & & & & & & & & & & 1.51 & $(1.27)$ & \\
\hline Estrategias de memorización & & & & & & & & & & -8.81 & $(1.41)$ & **** & -3.04 & (3.58) & \\
\hline Estrategias de memorización * Nivel socioeconómico IE & & & & & & & & & & & & & -2.61 & $(1.57)$ & \\
\hline Estrategias de control & & & & & & & & & & 5.09 & $(1.32)$ & *** & 2.18 & $(2.95)$ & \\
\hline Estrategias de control * Nivel socioeconómico IE & & & & & & & & & & & & & 1.22 & $(1.53)$ & \\
\hline Estrategias de comprensión & & & & & & & & & & 8.33 & (0.99) & **** & 9.34 & $(2.56)$ & *** \\
\hline Estrategias de comprensión * Nivel socioeconómico IE & & & & & & & & & & & & & -0.57 & $(1.20)$ & \\
\hline Estrategias de síntesis & & & & & & & & & & 6.64 & (1.39) & *** & 2.46 & (3.32) & \\
\hline Estrategias de síntesis * Nivel socioeconómico IE & & & & & & & & & & & & & 2.06 & $(1.35)$ & \\
\hline Estimulación docente & & & & & & & & & & 1.54 & $(1.39)$ & & 1.18 & (3.08) & \\
\hline Estimulación docente * Nivel socioeconómico IE & & & & & & & & & & & & & 0.27 & $(1.61)$ & \\
\hline Estrategias de estructuración & & & & & & & & & & -0.73 & $(1.38)$ & & 4.61 & (3.49) & \\
\hline Estrategias de estructuración * Nivel socioeconómico IE & & & & & & & & & & & & & -2.46 & $(1.71)$ & \\
\hline Relaciones profesor-alumno & & & & & & & & & & 2.61 & $(1.11)$ & * & 6.78 & $(3.81)$ & \\
\hline Relaciones profesor-alumno * Nivel socioeconómico IE & & & & & & & & & & & & & -1.99 & $(1.75)$ & \\
\hline Clima en el aula & & & & & & & & & & 5.66 & $(1.02)$ & **** & 15.06 & $(2.84)$ & **** \\
\hline Clima en el aula $*$ Nivel socioeconómico IE & & & & & & & & & & & & & -4.36 & $(1.22)$ & *** \\
\hline \multicolumn{16}{|l|}{ A nivel de la institución educativa } \\
\hline Intercepto & 354,06 & $(6,83)$ & **** & 223,37 & $(17,06)$ & **** & 227.00 & $(17.30)$ & **** & 230.66 & $(16.56)$ & **** & 230.27 & $(16.55)$ & *** \\
\hline Área (urbano) & & & & 30,59 & $(6,94)$ & ***** & 15.63 & $(6.87)$ & * & 18.00 & $(6.36)$ & *** & 19.21 & $(6.35)$ & *** \\
\hline Tipo de institución educativa (privado) & & & & 59,20 & $(7,19)$ & **** & 26.35 & $(8.35)$ & *** & 23.98 & $(7.79)$ & *** & 23.67 & $(7.80)$ & *** \\
\hline Tamaño de institución educativa & & & & 0,03 & $(0,01)$ & **** & 0.01 & $(0.01)$ & *** & 0.01 & $(0.00)$ & * & 0.01 & $(0.00)$ & * \\
\hline Estudiantes por docente & & & & $-0,83$ & $(0,45)$ & & -0.37 & $(0.44)$ & & -0.35 & $(0.40)$ & & -0.36 & $(0.39)$ & \\
\hline Terciles de nivel socioeconómico IE & & & & & & & 32.19 & $(4.01)$ & **** & 32.14 & $(3.77)$ & **** & 31.89 & $(3.88)$ & **** \\
\hline \multicolumn{16}{|l|}{ Descomposición de la varianza } \\
\hline A nivel del estudiante & \multicolumn{3}{|c|}{3882,96} & \multicolumn{3}{|c|}{3874,83} & & 1,28 & & & 8,05 & & & 0,65 & \\
\hline A nivel de la I.E. & & & & & & & & 3,66 & & & 4,97 & & & 1,79 & \\
\hline Proporción de varianza por institución educativa & & 56 & & & 76 & & &, 82 & & & 0,83 & & & 0,84 & \\
\hline Observaciones & & & & & & & & & & & & & & & \\
\hline Estudiantes & & 390 & & & 390 & & & 890 & & & 890 & & & 8890 & \\
\hline Instituciones educativas & & 40 & & & 40 & & & 240 & & & 240 & & & 240 & \\
\hline
\end{tabular}

Nota: ${ }^{*} * * \mathrm{p}<.001,{ }^{* *} \mathrm{p}<.01,{ }^{*} \mathrm{p}<.05$. Para el cálculo de los coeficientes fue usado el diseño muestral; el cálculo de los errores estándar se realizó usando el método de Balanced Repeated Replication (BRR). 
Los resultados de las encuestas de PISA sugieren que los estudiantes del tercil inferior tienen menores oportunidades; por ejemplo tienen menos actividades extracurriculares (de diverso tipo) y menos horas asignadas para el aprendizaje de ciencias y matemática que sus pares en los otros dos terciles (en comunicación el número de horas asignadas es similar entre terciles).

En cuanto a resultados, los estudiantes del tercil inferior son los que menores promedios tuvieron el 2009 en la prueba de comprensión de lectura, y los que menos subieron su puntaje desde el 2000. Prácticamente ningún estudiante en una institución educativa del tercil inferior se ubicó en el nivel de alto rendimiento en PISA. En muchas de las variables de recursos, procesos y resultados, la diferencia pareciera estar principalmente en la distancia entre el tercil inferior con el medio, más que del medio con el superior (por ejemplo el tercil medio fue el que más mejoró en la prueba PISA entre el 2000 y el 2009, aunque los del tercil superior mantienen los resultados más altos). Este tipo de resultado sugiere la importancia de la equidad como principio básico de las políticas educativas de los próximos años, orientándolas a elevar el promedio nacional al tiempo que se disminuyen las brechas de rendimiento entre grupos ligadas a nivel socioeconómico, etnicidad, zona de residencia (urbano versus rural) y gestión de la institución educativa (público versus privado). En un contexto de crecimiento de la cobertura escolar del nivel secundario, se vuelve especialmente relevante la atención a políticas de equidad (Tedesco y López, 2013). A continuación se discuten algunos resultados que podrían ayudar a desarrollar estas políticas vinculadas a comprensión de lectura.

El énfasis del presente estudio ha estado en variables que podrían ser influenciadas directamente desde el sistema educativo. No pretendemos aquí resumir todos los resultados que han sido presentados a lo largo del estudio, sino resaltar algunos que podrían parecer sorprendentes o prometedores para mejorar el rendimiento. Así, los estudiantes del tercil inferior son los que con mayor probabilidad reportan leer por placer, aunque cuentan con menos libros en casa; igualmente son los que más reportan utilizar bibliotecas. Tal vez esto pueda ser ligado al uso de computadoras y la comunicación de diferentes tipos vía internet, que es claramente más frecuente en estudiantes de las instituciones educativas del tercil superior. En general la disposición hacia la lectura es positiva en todos los sectores, lo cual es un primer elemento importante para obtener mejores resultados. Dada la disposición, la pregunta es cómo fomentar la lectura y sobre todo la comprensión en niveles complejos como los requeridos en PISA.

En cuanto a estrategias metacognitivas de los estudiantes y prácticas pedagógicas de los docentes, en algunos casos hay diferencias entre estudiantes por tercil socioeconómico, pero lo más trascendente son las asociaciones de estas con rendimiento en comprensión de lectura. Las asociaciones más fuertes con rendimiento fueron: 1) Memorización: los estudiantes que reportaron más estrategias de repetición del texto (por ejemplo "Leo el texto tantas veces que lo puedo repetir todo de memoria") tuvieron en general promedios más bajos; 2) Comprensión: Los estudiantes que reportaron estrategias para profundizar el significado de los textos (por ejemplo "Resumo el texto en mis propias palabras”) tuvieron en general promedios más altos; 3) Síntesis: Los estudiantes que reportan hacer sus propios resúmenes de la información (por ejemplo "Escribo un resumen. Luego, reviso que el resumen contenga la idea de cada párrafo, pues estas deben estar incluidas en el resumen") tuvieron mejores resultados; y 4) Clima en el aula: 
Los estudiantes que reportan mejor orden en el salón de clases (por ejemplo marcaron que "Los estudiantes no empiezan a trabajar sino hasta mucho tiempo después de haber comenzado la clase" no ocurría frecuentemente en su institución educativa) tuvieron mejor rendimiento.

Salvo para clima en el aula, no hubo interacciones entre las variables de intereses, estrategias o prácticas con rendimiento. En términos generales esto implica que el efecto de estas variables sería similar entre estudiantes de todos los terciles (en el caso de clima en el aula el beneficio resultó mayor en el tercil socioeconómico inferior). Estos resultados en líneas generales van en la misma dirección de lo que propone el DCN, presentado antes, así como balances de la literatura (Alverman y colaboradores, 2006).

Dado lo anterior, la implementación de estrategias para fomentar comprensión, síntesis y clima, y desalentar la memorización en la comprensión de lectura podría asociarse eventualmente a mejoras en el rendimiento, pero si estas se implementan universalmente no llevarían a una disminución de brechas por inequidad. La disminución de brechas requeriría en primer lugar trabajar de manera priorizada con grupos de estudiantes que se sabe tendrán dificultades en rendimiento; de acuerdo al análisis anterior y otros estudios de la misma UMC se trata de estudiantes rurales, con lengua materna indígena, en contextos de pobreza; en muchos casos un estudiante muestra dos o las tres características, ahondando el reto al mismo tiempo que lo facilita pues se trata de poblaciones estudiantiles en instituciones educativas públicasfácilmente identificables.

El estudio presentado aquí tiene algunas limitaciones. Todos los datos sobre estrategias, prácticas e intereses provienen del auto reporte de los estudiantes y en algunos casos de los directores de las instituciones educativas. Esto podría implicar sesgos, en el sentido que las respuestas podrían representar lo que se piensa es deseable y no datos objetivos. Sin embargo, este tipo de auto reporte es común para estos estudios en gran escala (más aún si son internacionales, como es el caso). Conseguir datos objetivos (por ejemplo de observación) es posible aunque altamente costoso. Pensamos que en lo anterior hay datos relevantes para continuar con una línea de investigación sobre procesos y resultados vinculados a comprensión de lectura en estudiantes peruanos a nivel secundario, que podría combinar diferentes técnicas de recojo de datos, como el auto reporte, la observación libre u objetiva, y el reporte de informantes calificados (por ejemplo docentes). Una de las contribuciones del presente estudio es que se enfoca en lectura durante la secundaria; la mayor parte de estudios y evaluaciones escolares disponibles en el Perú e incluso internacionalmente son de primaria (por ejemplo las evaluaciones censales en el Perú en $2^{\circ}$ grado de primaria, la evaluación del Laboratorio Latinoamericano de Evaluación de la Calidad de la Educación de la UNESCO en $4^{\circ}$ y $6^{\circ}$ de primaria (LLECE, 2008, 2010) y la evaluación PIRLS de lectura en $4^{\circ}$ de primaria.

Para tener influencia en políticas sin embargo, a menudo es conveniente contar con datos rigurosos que permitan establecer relaciones de causa y efecto, cosa que no es posible con un diseño como el empleado aquí. Para este fin, en el contexto científico actual se priorizan diseños que comparen el rendimiento de un grupo de tratamiento y uno de control, preferentemente con asignación aleatoria. Por ejemplo, McEwan (2015) publicó un metaanálisis de estudios experimentales en educación en países en desarrollo. $\mathrm{El}$ autor encuentra un efecto grande de las intervenciones orientadas al desarrollo profesional docente, un resultado coherente con lo sugerido aquí. Esta revisión incluyó algunos experimentos para incrementar el rendimiento en lectura, aunque su análisis se 
limitó a la educación primaria. Snilsteveit y colaboradores (2015) han publicado una revisión sistemática de la literatura que incluye una mayor gama de diseños de investigación (v. gr. diseños experimentales y cuasi experimentales). Ellos encuentran un impacto relativamente alto de intervenciones pedagógicas estructuradas (incluyendo diseño de contenidos, materiales educativos y trabajo con docentes).

Así, diseñar una intervención que se centre en elevar las estrategias metacognitivas de los estudiantes (v. gr. comprensión y síntesis, disminuyendo estrategias de memorización), mejorar el clima de aula y elevar el gusto por la lectura, y evalúe tal intervención con un diseño experimental parecería un siguiente paso lógico dados los resultados anteriores. Una intervención y evaluación de este tipo podrían ayudar a profundizar nuestra comprensión sobre cómo mejorar los niveles de comprensión de lectura de los estudiantes durante la secundaria, a la vez que permite contar con evidencia rigurosa del efecto de este tipo de intervenciones educativas.

\section{Agradecimientos}

El presente estudio fue encargado, financiado y comentado por la Unidad de Medición de la Calidad Educativa (UMC) del Ministerio de Educación de Perú. En particular, los autores agradecemos el apoyo y sugerencias de su Directora, Liliana Miranda. También agradecemos la asistencia de María Cristina Vásquez de GRADE en la revisión y mejora del presente documento, así como las sugerencias de dos revisores anónimos.

\section{Referencias}

Abadzi, H. (2011). Reading fluency measurements in EFA FTI partner countries: outcomes and improvement prospects. Washington, DC: World Bank.

Alverman, D., Simpson, M. y Fitzgerald, J. (2006). Teaching and learning in reading. En P. Alexander y P. Winne (Eds.), Handbook of educational psychology (pp. 427-256). Trenton, NJ: Lawrence Erlbaum Associates.

Benavides, M., León, J. y Etesse. (2014). Desigualdades educativas y segregación en el sistema educativo peruano. Una mirada comparativa de las pruebas PISA 2000 y 2009. Lima: GRADE.

Caro, D., Espinoza, G., Montané A. y Tam, M. (2004). Una aproximación a la alfabetización lectora de los estudiantes peruanos de 15 años: Resultados del Perú en la evaluación internacional PISA. Lima: UMC-Ministerio de Educación.

Cohen J. (1988). Statistical power analysis for the behavioral sciences. Nueva York: Routledge Academic

Cueto, S. (2007). Las evaluaciones nacionales e internacionales de rendimiento escolar en el Perú: balance y perspectivas. En Grupo de Análisis para el Desarrollo (Ed.), Investigación, políticas y desarrollo en el Perú (pp. 405-455). Lima: GRADE.

Ferrer, G. (2006). Sistemas de evaluación de aprendizajes en América Latina: balances y desafíos. Santiago: PREAL.

Hernández Padilla, E. y Bazán Ramírez, A. (2016). Efectos contextuales, socioeconómicos y culturales, sobre los resultados de México en Lectura en PISA 2009. REICE. Revista Iberoamericana sobre Calidad, Eficacia y Cambio en Educación, 14(2), 79-95. doi:10.15366/reice2016.14.2.005 
Linnakylä, P. y Välijärvi, J. (2006). Rendimiento de los estudiantes finlandeses en PISA: Las claves del éxito en lectura. Revista de Educación, número extraordinario, 227-235.

LLECE. (2008). Primer reporte. Segundo estudio regional comparativo y explicativo. Los aprendizajes de los estudiantes de América Latina y el Caribe. Santiago: UNESCO.

LLECE. (2010). Factores asociados al logro cognitivo de los estudiantes de América Latina y el Caribe. Santiago: UNESCO

McEwan, P. J. (2015). Improving learning in primary schools of developing countries: A metaanalysis of randomized experiments. Review of Educational Research, 85(3), 353-394. doi:10.3102/0034654314553127.

Ministerio de Educación. (2006). Guía para el desarrollo de capacidades comunicativas. Lima: MED.

Ministerio de Educación. (2008a). Diseño curricular nacional de educación básica regular. Lima: MED.

Ministerio de Educación. (s/f). Orientaciones específicas para el plan lector nivel secundaria. Recuperado de http://www.oei.es/

National Reading Panel. (2000). Teaching children to read: an evidence-based assessment of the scientific research literature on reading and its implications for reading instruction. Recuperado de https://www.nichd.nih.gov/

OECD. (2009). PISA 2009. Assessment framework - key competencies in reading, mathematics and science. París: OECD.

OECD. (2009). PISA 2006 technical report. París: OECD.

OECD. (2010). PISA 2009 results: what students know and can do - student performance in reading, mathematics and science (Volume 1). París: OECD.

Paris, S., Morrison, F. y Miller, K. (2006). Academic pathways from preschool through elementary school. En P. Alexander y P. Winne (Eds.), Handbook of educational psychology (pp. 61-87). Trenton, NJ: Lawrence Erlbaum Associates

Raudenbush, S. y Bryk, A. S. (2002). Hierarchical linear models: Applications and data analysis methods ( $2^{\circ} e d$.). Newbury Park, CA: Sage.

Rodríguez-Valls, F. (2010). Los procesos de calidad en la enseñanza de la lectura, origen de un saber democrático y participativo. REICE. Revista Iberoamericana sobre Calidad, Eficacia y Cambio en Educación, 8(5), 119-132.

Royston, P. (2004). Multiple imputation of missing values. Stata Journal, 4(3), 227-241.

Schubert, F. y Becker, R. (2010). Social inequality of reading literacy. Research in Social Stratification and Mobility, 28(1), 109-133. doi:10.1016/j.rssm.2009.12.007

Schwabe, F., McElvany, N., y Trendtel, M. (2014). The school age gender gap in reading achievement: examining the influences of item format and intrinsic reading motivation. Reading Research Quarterly, 50(2), 219-232. doi:10.1002/rrq.92.

Snilstveit, B., Stevenson, J., Phillips, D., Vojtkova, M., Gallagher, E., Schmidt, T., Jobse, H., Geelen, M., Pastorello, M. G. y Eyers, J. (2015). Interventions for improving learning outcomes and access to education in low- and middle-income countries: a systematic review. Recuperado de http://www.3ieimpact.org/

Tedesco, J. C. y López, N. (2013). Diez años después: Comentarios tras una relectura del artículo "Algunos dilemas de la educación secundaria en América Latina”. REICE. Revista Iberoamericana sobre Calidad, Eficacia y Cambio en Educación, 11(2), 10-32. 
Tiana Ferrer, A. (2011). ¿Y después de PISA qué? REICE. Revista Iberoamericana sobre Calidad, Eficacia y Cambio en Educación, 9(1), 3-5.

\section{Anexo 1. Índices utilizados}

A continuación se presentan las definiciones de los índices utilizados en el análisis:

Índice de placer por la lectura fue construido usando los ítems: Leer es uno de mis pasatiempos favoritos; Me gusta hablar sobre los libros con otras personas; Me alegra recibir libros como regalo; Disfruto visitando librerías y bibliotecas; Leo solamente para obtener la información que necesito. Se usaron los valores de la escala (de 1 a 4), estandarizando el promedio nacional en o y la desviación estándar en 1, haciendo el cambio de signo en los ítems que fuera necesario. La confiabilidad de la escala resultante fue de 0.66 para el 2000 y 0.69 para el 2009 (Alpha de Cronbach). Para el índice de placer por la lectura se encontró un porcentaje de observaciones perdidas de $15 \%$ para el 2000 y de $6 \%$ para el 2009 .

El índice de uso de estrategias de memorización fue construido usando los ítems Trato de memorizar todo lo que está en el texto; Trato de memorizar tantos detalles como sea posible; Leo el texto tantas veces que lo puedo repetir todo de memoria; Leo el texto varias veces. Se usaron los valores de la escala (de 1 a 4 ) y estandarizando el promedio nacional a 0 y la desviación estándar a 1, haciendo el cambio de signo en los ítems que fuera necesario. La confiabilidad de la escala resultante fue de 0.62 (Alpha de Cronbach). Para el índice de uso de estrategias de memorización se encontró un porcentaje de observaciones perdidas de 5\% para el 2009.

El índice de uso de estrategias de elaboración fue construido con base en los ítems: Trato de relacionar la nueva información al conocimiento adquirido previamente en otros cursos; Analizo de qué forma esta información podría ser de utilidad fuera del colegio; Trato de comprender mejor la información relacionándola con mis propias experiencias; Analizo cómo el texto encaja con lo que ocurre en la vida real. Se usaron los valores de la escala (de 1 a 4) y estandarizando el promedio nacional a 0 y la desviación estándar a 1, haciendo el cambio de signo en los ítems que fuera necesario. La confiabilidad de la escala resultante fue de 0.68 (Alpha de Cronbach). Para el índice de uso de estrategias de elaboración se encontró un porcentaje de observaciones perdidas de 5\% para el 2009.

El índice de uso de estrategias de control fue construido con base en los ítems: Comienzo por analizar lo que realmente necesito aprender; Reviso si entiendo lo que he leído; Trato de que darme cuenta de qué conceptos aún no he logrado realmente entender; Me aseguro de recordar los puntos más importantes del texto; Cuando no entiendo algo, busco información adicional para esclarecer mis dudas. Se usaron los valores de la escala (de 1 a 4), estandarizando el promedio nacional a 0 y la desviación estándar a 1, haciendo el cambio de signo en los ítems que fuera necesario. La confiabilidad de la escala resultante fue de 0.69 (Alpha de Cronbach). Para el índice de uso de estrategias de control se encontró un porcentaje de observaciones perdidas de 5\% para el 2009.

El índice de uso de estrategias de compresión y recuperación de información se construyó con base en los ítems: Me concentro en las partes del texto que son fáciles de comprender; Leo rápidamente el texto dos veces; Después de leer el texto, discuto su contenido con otras personas; Subrayo las partes importantes del texto; Resumo el texto en mis propias palabras; Leo el texto en voz alta a otra persona. El índice fue construido usando los valores de la escala (de 1 a 6 ), 
estandarizando el promedio nacional a 0 y la desviación estándar a 1, haciendo el cambio de signo en los ítems que fuera necesario. La confiabilidad de la escala resultante fue de 0.73 (Alpha de Cronbach). Para el índice de uso de estrategias de compresión y recuperación de información se encontró un porcentaje de observaciones perdidas de $13 \%$ para el 2009.

El índice de uso de estrategias de síntesis fue construido con base en los ítems: Escribo un resumen, luego reviso que el resumen contenga la idea de cada párrafo, pues estas deben estar incluidas en el resumen; Trato de copiar con exactitud tantas oraciones como sea posible; Antes de escribir el resumen, leo el texto tantas veces como sea posible; Compruebo cuidadosamente que los hechos más importantes del texto estén incluidos en el resumen; Leo a través del texto, subrayando las oraciones más importantes. Luego las escribo en mis propias palabras a manera de resumen. Se usaron los valores de la escala (de 1 a 6), estandarizando el promedio nacional a 0 y la desviación estándar a 1, haciendo el cambio de signo en los ítems que fuera necesario. La confiabilidad de la escala resultante fue de 0.74 (Alpha de Cronbach). Para el índice de uso de estrategias de síntesis se encontró un porcentaje de observaciones perdidas de 13\% para el 2009.

El índice de estimulación docente fue construido con base en los ítems: El profesor pide a los estudiantes que expliquen el significado del texto leído; El profesor les hace preguntas que reta a los estudiantes a comprender mejor un texto; El profesor da suficientes tiempo a los estudiantes para pensar su respuesta; El profesor recomienda un libro o un autor para leer; El profesor motiva a sus estudiantes a expresar su opinión sobre un texto; El profesor ayuda a los estudiantes a relacionar las historias que leen con lo que les pasa en sus vidas; El profesor muestra a sus estudiantes cómo la información de los textos se basa en temas que ya conocen. Se usaron los valores de la escala (de 1 a 4), estandarizando el promedio nacional a o y la desviación estándar a 1, haciendo el cambio de signo en los ítems que fuera necesario. La confiabilidad de la escala resultante fue de 0.79 (Alpha de Cronbach). Para el índice de estimulación docente se encontró un porcentaje de observaciones perdidas de 7\% para el 2009.

El índice de uso de estrategias de estructuración fue construido con base en los ítems: El profesor explica con anticipación lo que se espera de sus estudiantes; El profesor se asegura de que sus estudiantes estén concentrados mientras hacen sus tareas de lectura; El profesor comenta el trabajo de sus estudiantes después de que éstos terminan su tarea de lectura; El profesor les indica por adelantado a sus estudiantes cómo van a ser evaluados sus trabajos; El profesor pregunta si todos han entendido cómo hacer sus tareas de lectura; El profesor califica los trabajos de los estudiantes; El profesor les da oportunidad a los estudiantes de preguntas sobre sus tareas de lectura; El profesor hace preguntas que motivan a sus estudiantes a participar activamente; El profesor les dices a sus estudiantes cuán bien realizaron sus tareas de lectura inmediatamente después de terminarlas. Se usaron los valores de la escala (de 1 a 4), estandarizando el promedio nacional a o y la desviación estándar a 1, haciendo el cambio de signo en los ítems que fuera necesario. La confiabilidad de la escala resultante fue de 0,84 (Alpha de Cronbach). Para el índice de uso de estrategias de estructuración se encontró un porcentaje de observaciones perdidas de $9 \%$ para el 2009.

El índice de relaciones docente-alumno fue construido con base en los ítems: Me llevo bien con la mayoría de mis profesores; La mayoría de mis profesores están interesados en mi bienestar; La mayoría de mis profesores escuchan lo que tengo que decir; Si necesito 
ayuda adicional, la puedo recibir de mis profesores; La mayoría de mis profesores me tratan de manera justa. Se usaron los valores de la escala (de 1 a 4), estandarizando el promedio nacional a 0 y la desviación estándar a 1, haciendo el cambio de signo en los ítems que fuera necesario. La confiabilidad de la escala resultante fue de 0,74 para el 2000 y 0.78 para el 2009 (Alpha de Cronbach). Para el índice de relaciones profesoralumno se encontró un porcentaje de observaciones perdidas de $7 \%$ para el 2000 y de $5 \%$ para el 2009.

El índice de actividades extracurriculares se construyó con base en los ítems: Banda, orquesta o coro; Teatro escolar o musicales; Anuario, periódico o revista; Voluntariado o actividades de servicio (por ejemplo: visitas a hospitales, sembrar árboles, etc.); Club de lectura; Club de debate o actividades de debate; Concursos de idiomas extranjeros, matemática o ciencia; Club de estudio (por ejemplo: club de ciencias, club de matemática, club de ajedrez, etc.); Club de arte o actividades artísticas; Club de deportes o actividades deportivas; Charlas y/o seminarios (por ejemplo: expositores tales como escritores o periodistas); Colaboración con bibliotecas locales (por ejemplo: convenios, para que los estudiantes puedan visitar y prestarse libros); Colaboración con periódicos locales (por ejemplo: estudiantes que participan como corresponsales periodísticos). El índice fue construido usando los valores de 1 y o de la variable, estandarizando el promedio nacional a 0 y la desviación estándar a 1, haciendo el cambio de signo en los ítems que fuera necesario. La confiabilidad de la escala resultante fue de 0,67 (Alpha de Cronbach). Para el índice de actividades extracurriculares se encontró un porcentaje de observaciones perdidas de 10\% para el 2009.

\section{Anexo 2. Variables incluidas en el análisis de regresión multinivel}

Variable dependiente:

- Lectura: Puntajes promedio en la prueba de comprensión lectora PISA.

Variables independientes a nivel del estudiante o su familia:

- Género: variable dicotómica que toma el valor de 1 si el estudiante es mujer y o si es hombre.

- Lengua materna: variable dicotómica que toma el valor de 1 si es castellano y o en otro caso.

- Estructura Familiar: esta variable puede tener tres valores: familia de padre/madre soltera si el estudiante vive solo con uno de los siguientes: madre, padre, guardián hombre o guardián mujer; familia nuclear cuando el estudiante vive con ambos padres en el hogar, incluyendo en este caso padres adoptivos; y familia mixta en cualquier otro caso. Para capturarla se han incluido en el modelo dos variables dicotómicas.

- Nivel socioeconómico: índice sobre el nivel socioeconómico del hogar del niño compuesto por la ocupación de los padres, su nivel educativo y el índice de posesiones en el hogar. 
- Asistió a educación inicial: variable dicotómica que toma el valor de 1 si el estudiante asistió en algún momento a educación inicial y o en otro caso.

- Grado para la edad: es una variable que se codifica con o si el estudiante está en el grado que le corresponde de acuerdo a la normativa y valores positivos o negativos en años de adelanto o atraso escolar según fuera el caso.

- Horas de clase en comunicación: variable continua, la cual se obtuvo del reporte de los estudiantes.

- Lectura por placer: índice de placer por la lectura construido a partir de un promedio simple de distintos ítems del cuestionario del estudiante.

- Estrategias de memorización: índice de memorización construido a partir de un promedio simple de distintos ítems del cuestionario del estudiante.

- Estrategias de control: índice de control construido a partir de un promedio simple de distintos ítems del cuestionario del estudiante.

- Estrategias de comprensión y recuperación de información: índice de compresión y recuperación de información construido a partir de un promedio simple de distintos ítems del cuestionario del estudiante.

- Estrategias de sintesis: índice de síntesis construido a partir de un promedio simple de distintos ítems del cuestionario del estudiante.

- Estimulación docente: índice de estimulación docente construido a partir de un promedio simple de distintos ítems del cuestionario del estudiante.

- Estrategias de estructuración: índice de estructuración construido a partir de un promedio simple de distintos ítems del cuestionario del estudiante.

- Relaciones profesor-alumno: índice de relaciones profesor-alumno construido a partir de un promedio simple de distintos ítems del cuestionario del estudiante.

- Clima en el aula: índice de clima de disciplina en el aula construido a partir de un promedio simple de distintos ítems del cuestionario del estudiante.

Variables independientes a nivel de la Institución educativa:

- Area: variable dicotómica que toma el valor de 1 si la I.E. se encuentra en área rural y o si se encuentra en área urbana (rural fue definido por el propio director, refiriéndose a la ubicación de la institución educativa en una comunidad de menos de 3.000 personas).

- Tipo de institución educativa: variable dicotómica que toma el valor de o si la I.E. es pública y 1 si es privada o de gestión privada (reporte del director).

- Tamaño de institución educativa: variable que indica el número total de estudiantes en la I.E (reporte del director).

- Estudiantes por docente: variable que indica el número de estudiantes por cada docente en la I.E (construida con base en el reporte del director). 
- Nivel socioeconómico promedio de la IE: se usó la variable creada para el estudio, presentada anteriormente, con valores 3 para el tercil superior, 2 para el medio y 1 para el inferior.

\section{Anexo 3. Análisis multivariado de estrategias y prácticas pedagógicas individualmente con lectura}

Para definir cuáles de las variables de prácticas y estrategias pedagógicas se incluirían finalmente en el análisis multivariado se corrió una regresión multinivel ajustando por características individuales del individuo y la institución educativa (modelo 3 de la Tabla 6). Solo se incluyeron las variables con coeficiente significativo en el análisis final. Los coeficientes simples se presentan en el Cuadro siguiente.

Tabla 1. Coeficiente de variables de estrategias pedagógicas

\begin{tabular}{lrc}
\hline & \multicolumn{2}{c}{ RENDIMIENTO EN COMPRENSIÓN } \\
LECTORA
\end{tabular}

Nota: $* * * \mathrm{p}<.01, * * \mathrm{p}<.05, * \mathrm{p}<.10$

En todos los casos se controló por las variables individuales (género, lengua, estructura familiar, nivel socioeconómico, asistió a educación inicial, grado para la edad y horas de clase en comunicación) y de la institución educativa (área, tipo de institución educativa, tamaño de institución educativa, estudiantes por docente y terciles de nivel socioeconómico)

Para el cálculo de los coeficientes fue usado el diseño muestral y el cálculo de los errores estándar se realizó usando el método de Balanced Repeated Replication (BRR).

De acuerdo a lo anterior, no se incluyó en el modelo final el índice de estrategias de elaboración. No se incluyeron en el análisis otras variables relacionadas a las estrategias y prácticas pedagógicas que en los análisis preliminares no tuvieron asociación estadísticamente significativa con rendimiento.

\section{Breve CV de los autores}

\section{Santiago Cueto}

Licenciado en Psicología Educacional por la Pontificia Universidad Católica del Perú (PUCP) y Doctor en Psicología Educacional por la Universidad de Indiana. Ha sido investigador visitante de las Universidades de California en Davis, Pensilvania y Oxford. Actualmente es Director de Investigación de GRADE y representante por el Perú para 
el estudio internacional Young Lives. Es miembro del Consejo Nacional de Educación y profesor principal del Departamento de Psicología de la PUCP. Fue Presidente de la Sociedad de Investigación Educativa Peruana. Sus principales áreas de interés son educación y desarrollo humano, en particular en contextos de pobreza. En el 2003, en la conferencia anual del Global Development Network, recibió la medalla a la mejor investigación en la categoría Educación, Conocimiento y Tecnología. El 2010 el Colegio de Psicólogos del Perú le otorgó el Premio Nacional de Psicología. Email: scueto@grade.org.pe

\section{Juan León}

Juan es candidato a PhD en Teorías y Políticas Educativas y Educación Internacional Comparada por la Universidad Estatal de Pennsylvania. Es Bachiller en Ciencias Sociales con mención en Economía por la Pontificia Universidad Católica del Perú. Actualmente es Investigador Asociado de GRADE del área Educación y aprendizajes. Sus temas de investigación abarcan los efectos escolares, la eficacia escolar, los efectos de la escolaridad, la educación bilingüe, las inequidades educativas, el desarrollo infantil y la evaluación de impacto. Email: jleon@grade.org.pe

\section{Ismael G. Muñoz}

Ismael es estudiante de doctorado en Teorías y Políticas Educativas y Educación Internacional Comparada en la Universidad Estatal de Pennsylvania. Es Magister en Economía Internacional y del Desarrollo por la Escuela de Economía de Louvain Universidad de Namur (Bélgica), y Bachiller en Economía por la Pontificia Universidad Católica del Perú. Se ha desempeñado como investigador en el Ministerio de Educación del Perú y como investigador asistente en GRADE. Sus principales temas de investigación son el desarrollo infantil temprano, los efectos de la educación, las inequidades educativas y la evaluación de impacto de programas sociales. Email: igm104@psu.edu

\section{Elizabeth Rosales}

Elizabeth es candidata a PhD en Estudios Curriculares y Desarrollo Docente por la Universidad de Toronto. Es Licenciada en Psicología Educacional por la Pontificia Universidad Católica del Perú y Magíster en Educación por la Universidad de Toronto. Su tesis de licenciatura fue premiada por la Sociedad de Investigación Educativa Peruana en el 2010. Trabajó en GRADE como investigadora asistente durante el 2010-2012. Sus temas de investigación incluyen la formación docente, la educación continua de maestros, y los procesos de aprendizaje de docentes. Email: elizabeth.rosales@mail.utoronto.ca 